\title{
Tying and Freebies in Two-Sided Markets Preliminary and Incomplete*
}

\author{
Andrea Amelio ${ }^{\dagger} \quad$ Bruno Jullien ${ }^{\ddagger}$
}

March 2007

\begin{abstract}
In two-sided markets where platforms are constrained to set nonnegative prices, we study the effect of tying and pure bundling. Bundling can be deployed by platforms as a tool to introduce implicit subsidies on one side of the market in order to solve the usual coordination failure in a two-sided market framework. A multi-product monopoly platform uses bundles to raise participation on both sides, which benefits consumers. In a duopoly context, bundles have also a strategic effect on the level of competition. Contrary to the monopoly case, tying may not be ex-post and/or ex-ante optimal for a contested platform. Moreover, the competing platform benefits from it if the equilibrium implicit subsidy is large enough. The impact on consumers surplus and total welfare depends on the extent of asymmetry in externalities between the two sides, with a negative effect if there is little symmetry, and a positive effect with strong asymmetry.
\end{abstract}

\section{Introduction}

One key issue for two-sided platforms is that the need to coordinate consumers on an efficient allocation may require subsidizing the participation of some consumers. Based on the initial work of Caillaud and Jullien (2003), Rochet and Tirole (2003) and Armstrong (2005), the literature has emphasized the role of the price structure in solving coordination problems. Caillaud and Jullien (2003) and Armstrong (2005) show that platforms may set "negative prices" on one side in order to enhance participation. However, a direct implementation of negative prices is not always a viable option

\footnotetext{
${ }^{*}$ The authors thank Jean-Charles Rochet, David Evans, Mark Armstrong and Michael Katz for useful discussions and comments, as well as participants to the IDEI Conference on Software and Internet Industries.

${ }^{\dagger}$ University of Toulouse (GREMAQ, Université de Toulouse), andrea.amelio@univtlse1.fr

${ }^{\ddagger}$ University of Toulouse (GREMAQ and IDEI), bjullien@cict.fr
} 
to guarantee sufficient participation to the platform. Adverse selection and opportunistic behavior of agents can be two main risks that platforms could face by offering a direct monetary transfer to agents. As emphasized in Jullien (2005), when a platform is constrained to set non-negative prices or simply perceives a monetary transfer as too risky, one alternative is to rely on bundles designed to target some customers.

In the paper, we develop a model where a platform constrained to set non-negative prices ties the sales of another good with the access to the platform as a way to relax the non-negativity constraint. By giving away the bundle for free or at a discounted price, conditional on participation to one side, the platform eventually implements implicit negative subscription prices in a context where monetary subsidy would be inefficient or could generate opportunistic behaviors.

The concept of two-sided market refers to a specific instance of networks where the services are used by two distinct groups of customers interacting. This includes, among other activities, intermediation, credit card, medias, computer operating systems, video games, shopping malls or yellow pages. ${ }^{1}$ In these markets, tying is a widespread phenomenon and may take several forms. One form corresponds to the practice of offering gifts along with the service, as a magazine offering a DVD with its paper version. Another form, illustrated by the case of the Windows Media Player, consists in bundling a monopoly good with a complementary competitive two-sided good. ${ }^{2}$ Last but not least, a widespread practice among web portals like Yahoo or Google consists in offering for free a large bundle of services to one side of the market. More importantly, addressing the fact that in several cases the bundle is offered for free to consumers can be difficult and can pose problems when judging anti-trust cases. This paper offers a framework in which this issue can be addressed.

In the paper, the rationale for tying differs from entry deterrence purposes emphasized by Whinston (1990), and from price discrimination motives as developed in Adams and Yellen (1976) or Schmalensee (1984). The only purpose of tying is an attempt to stimulate demand on one side in order to increase the membership value and profit on the other side. The advantage is that the platform may avoid attracting undesirable customers by tying the platform service with a good of particular interest for the targeted side. Clearly for such a strategy to be effective, there must be some advantage of in kind payments over money subsidies. Thus, the best candidate is a complementary good. A free parking at a shopping mall is only interesting for customers intending to shop, or Google desktop-bar is only

\footnotetext{
${ }^{1}$ See Evans (2003a), Evans (2003b) and Rochet and Tirole (2005), Rochet and Tirole (2003) for more examples.

${ }^{2}$ See Choi (2004).
} 
interesting for web-surfers. However, since the purpose is to give subsidies to some participants, even an independent good can be used provided that its demand is related with the demand of two-sided services. For instance, magazine offering a CD or a DVD can choose to target a particular population. To this extent, the theory developed in the paper illustrates an instance of second-degree price discrimination implemented through tying which confirms the more general idea that price discrimination may help a network to coordinate the customers' participation and thereby be welfare improving (See Jullien (2001)).

The tying strategy is studied in the context of a monopolistic and a duopolistic two-sided market, similar to Armstrong (2005), where a participant on one side derives a positive externality increasing with the level of participation on the other side. In the duopoly case, platforms are horizontally differentiated on both sides, and agents register to one platform only (single-homing). We allow one platform to deploy mixed bundling (which reduces to tying in our model) and pure bundling strategies and analyze their impact on the allocation, as well as on the consumer surplus, the platforms' profits and the total welfare. In our model, the demand for the tied good is homogeneous among members of one side so that there is no price discrimination possibility within sides. In order to emphasize the subsidy aspect, we focus mostly on the case of tying where it is the sole effect at work. We then extend the analysis to pure bundling.

In a monopolistic context, when the platform has some market power on the tied good market, it uses a tying strategy and sells the good unbundled and bundled. The only reason to sell a bundle is to offer a participation subsidy. The subscription is offered for free, thus the bundle is sold at a discounted price. When the platform has no market power and the good is sold on a competitive market, it can still sell a pure bundle at price below cost. We show that all consumers benefit from these practices, since the enhanced participation of one side raises the perceived quality of the service on the other side.

In a duopolistic context, we account for strategic effects as the use of bundles affects the competitor's behavior. In a one-sided context such as ours, Whinston (1990) shows that mixed bundling is neutral while pure bundling reduces the equilibrium profit of all platforms and thus may result in entry deterrence. Since it implies a lower price of the platform service on the targeted side, tying is not neutral to profit. Moreover the strategic effects differ substantially because in our model the service is offered for free on the side subject to tying and profits are obtained by charging the other side.

A key point is that tying occurs on the side where the platform is subsidizing participation. Intuition may be misleading unless one realizes that 
the subsidized size should be considered only as the "input" sold to the other side. Increasing the subsidy through tying has two effects. First, for the tying platform, enhanced participation of one side gives a "quality" advantage on the other side and allows to raise the price for a given participation. But a by-product is such that it increases the opportunity cost of selling on the profitable side. The reason is that more sales on the profitable side implies more sales at a loss on the subsidized side. The opportunity cost of selling on the profitable side then accounts for the loss generated on the subsidized side, and increases with tying. Thus tying on one side also affects the intensity of competition on the other side.

The impact of tying on the behaviour of the platform on the profitable side has thus to be analyzed as the combined effects of increasing the perceived quality and increasing the (opportunity) cost. There is then a demand shifting effect associated with higher quality, and a competition softening effect associated with higher cost. The impact on equilibrium profits is then ambiguous and due to softened competition, the competitor's profit may increase when one platform uses tying. Similarly the tying platform may obtain less profits.

The impact on consumers is also ambiguous. Consumers on the subsidized side always benefit from a larger subsidy, but consumers on the profitable side may benefit or not depending on which of the two effects dominates. The main conclusions are: $i$ ) when the two sides evaluate the participation of each other in a symmetric way, total consumer surplus and total welfare decrease with tying; ii) when the subsidy is given to consumers who do not value the participation of the other side, then consumer surplus increases on both sides. But in any case some consumers are hurt, who may be clients of either platforms.

We then compare pure bundling and tying, where we use the usual feature that pure bundling modifies the opportunity cost of selling to the subsidized side.

Despite the importance of tying in two-sided markets, there has been little contribution to this issue. Rochet and Tirole (2005) analyze the practice of tying credit and debit card on the merchant side of the payment card market, and show that this results in a more efficient setting of interchangefees. They share with us the conclusion that tying may enhance efficiency by inducing a better coordination between the various sides. Choi (2004) analyzes a situation inspired by the Windows Media Players where one or both sides multi-home and the tied good is essential to participation to the platform. He shows that even if foreclosure may arise the welfare implications are ambiguous. In a very preliminary work, Farhi and Hagiu (2004) analyze the strategic implications of pure bundling focusing on the fact that it reduces the perceived marginal cost of selling to the targeted side. As in Choi (2004), their model assumes multi-homing on one side. They find that in some cases tying may soften competition and raise profit of both plat- 
forms. We corroborate this finding for mixed and pure bundle with single homing. Although the key mechanisms are different, our paper shares the fact that bundling results in enhanced participation on one side.

The paper is organized as follows. In Section 2 we set up a two-sided monopolistic framework. constraining prices to be non-negative. We define sufficient conditions to have a zero price in equilibrium and we study the impact of relaxing the non-negativity constraint by means of tying. Section 3 extends the analysis to a duopolistic framework with tying. Section 4 compares pure bundling and tying. Section 5 allows the bundled good to have a negative social value and discusses pure bundling in this context.

\section{A monopoly platform}

\section{$2.1 \quad$ Two-sided market and negative prices}

Consider a platform serving two groups of agents, denoted by 1 and 2, each of total size 1 . The platform incurs a cost $f_{i}$ for each agent subscribing to side $i$. Every agent of each group cares about the total number of agents in the other group. Anticipating on the duopolistic model and for conciseness, we assume a Hotelling model on each side where the firm is located at the extreme of the interval. ${ }^{3}$ Thus agents on each side are indexed by a parameter $x$ that is uniformly distributed on $[0,1]$. If the platform attracts $n_{-i}$ on the other side, the utility of the agents of side $i$ located at $x$ is $u_{i}=v_{i}-t_{i} x+\alpha_{i} n_{-i}-p_{i}$, where $v_{i}>f_{i}$ is the intrinsic valuation of participation, $\alpha_{i} \geq 0$ is the benefit of interacting with every agent belonging to the other group and $x$ is the heterogeneity parameter. In the monopoly section we focus on cases where the market is not covered and demand is differentiable. Thus consumers on side $i$ buy the service of the platform if $x<x_{i}$, where $0<x_{i}<1$. Defining $D_{i}(h)=\left(v_{i}-h\right) / t_{i}$, the numbers of agents $n_{1}$ and $n_{2}$ of each group participating is solution of the following system of equations:

$$
n_{1}=D_{1}\left(p_{1}-\alpha_{1} n_{2}\right) \text { and } n_{2}=D_{2}\left(p_{2}-\alpha_{2} n_{1}\right) .
$$

We assume that:

Assumption $1 \Delta=4 t_{1} t_{2}-\left(\alpha_{1}+\alpha_{2}\right)^{2}>0$.

For the purpose of the presentation, we also define $\Gamma=t_{1} t_{2}-\alpha_{1} \alpha_{2}$ which by Assumption 1 is positive and $\sigma=\alpha_{2}-\alpha_{1}$ which captures the asymmetry in network externalities. Notice that $\Delta=4 \Gamma-\sigma^{2}$.

As said above, we assume that $n_{i}<1$ in all equilibria. Under these assumptions, the relationship between the prices $\left(p_{1}, p_{2}\right)$ and the allocation

\footnotetext{
${ }^{3}$ The conclusions of the monopoly section extend easily to more general demand functions.
} 
$\left(n_{1}, n_{2}\right)$ is one-to-one, and all the profit maximization programs are concave. More precisely we have:

$$
n_{1}=\frac{t_{2}\left(v_{1}-p_{1}\right)+\alpha_{1}\left(v_{2}-p_{2}\right)}{\Gamma} \text { and } n_{2}=\frac{t_{1}\left(v_{2}-p_{2}\right)+\alpha_{2}\left(v_{1}-p_{1}\right)}{\Gamma} \text {. }
$$

Since our objective is to study how the platform can circumvent the impossibility to set a negative price on one side, price $p_{1}$ is constrained to be non-negative, while price $p_{2}$ is free. One can view the situation on side 1 as one where there is a large population that is indistinguishable from the targeted population and that may claim the service while not really using it if the price is negative. This creates a kink in demand at a zero price, as undesirable customers start to buy. If the kink is steep enough, the platform will not set a negative price.

The platform problem is then

$$
\begin{aligned}
& \Pi^{*}=\max _{p_{1}, p_{2}}\left(p_{1}-f_{1}\right) n_{1}+\left(p_{2}-f_{2}\right) n_{2} \\
& \text { s.t. } p_{1} \geq 0 \text { and }(1) .
\end{aligned}
$$

When the non-negative price constraint is not binding, standard monopoly pricing formulas are obtained on each side, where the relevant cost is the opportunity cost $f_{i}-\alpha_{-i} n_{-i}$ of attracting one agent of side $i$ instead of $f_{i}$. This opportunity cost reflects the fact that one additional customer on side $i$ allows raising the price by $\alpha_{-i}$ for the $n_{-i}$ agents of the other side. We thus have $p_{i}-f_{i}+\alpha_{-i} n_{-i}=-D_{i} / D_{i}^{\prime}$ on each side. ${ }^{4}$ In our linear case, the price on side 1 is:

$$
p_{1}^{*}=f_{1}+\frac{\left(v_{1}-f_{1}\right)\left(2 \Gamma-\alpha_{2} \sigma\right)+t_{1} \sigma\left(v_{2}-f_{2}\right)}{\Delta}
$$

and the allocation is:

$$
n_{i}^{*}=\frac{2 t_{-i}\left(v_{i}-f_{i}\right)+\left(\alpha_{1}+\alpha_{2}\right)\left(v_{-i}-f_{-i}\right)}{\Delta} .
$$

Following from the formula above, consumers of side 1 can be offered the service at a price below cost. The platform could (if possible) also set a negative price and thus offer a direct subsidy. From now on we assume that this is the case:

Assumption $2 p_{1}^{*}<0$.

\footnotetext{
${ }^{4}$ See Armstrong (2005) or Jullien (2005).
} 
Under Assumption 2, the non-negativity constraint binds and the price on side 1 is $p_{1}=0$. This only occurs when the side 1 is the low externality side. Indeed one may verify that Assumptions 1 and 2 imply that $\sigma=$ $\alpha_{2}-\alpha_{1}>0$. Notice that our assumptions also imply that $p_{2}>0$. The reason is that profits are positive since the platform could sell positive quantities at prices equal to costs. The optimal price $p_{2}$ satisfies the following condition (See Appendix for the full derivation):

$$
p_{2}-f_{2}+\alpha_{1}\left(f_{1}-\alpha_{2} n_{2}\right) D_{1}^{\prime}=-\frac{n_{2}}{D_{2}^{\prime}},
$$

where $D_{i}^{\prime}=-\frac{1}{t_{i}}$ is the slope of the demand of one side at fixed participation of the other side.

One can recognize a standard monopoly pricing formula for side 2 adjusted for the impact on side 1 . First notice that the opportunity cost of one more sale on side 1 is $f_{1}-\alpha_{2} n_{2}$, as above, since the price is flexible on side 2 and can be adjusted to maintain demand. However, given that $p_{1}$ is now fixed at zero, attracting one more individual on side 2 cannot be accommodated by changing $p_{1}$. The effect is now to raise the sales on side 1 by $-\alpha_{1} D_{1}^{\prime}$.

The fact that the price constraint binds (Assumption 2) implies also that $\alpha_{2} n_{2}>f_{1}$, which expresses that there must be a recoupment on side 2 for any member of side 1 . Indeed, there is no point of servicing side 1 at a loss unless the externality created for side 2 is larger than the cost on side 1 .

For a given level of $n_{1}$, equilibrium conditions imply that the price $p_{2}$ is smaller than in the unconstrained case. Thus, controlling for $n_{1}$, the platform sells more on side 2 in the constrained case. The reason is that the fact that an increase in side 2 sales raises the demand on side 1 tends to limit the incentive to raise sales on side 2 when the margin is negative on side 1 . Although raising $p_{1}$ is detrimental as it reduces $n_{1}$ and forces to reduce $p_{2}$, it also reduces the loss on side 1 and thus mitigates this effect. A second effect is that as $n_{1}$ decreases the perceived quality and thus the demand on side 2 decreases.

In our linear set-up, price on side 2 in the constrained case is $p_{2}^{0}=\frac{t_{1}\left(v_{2}+f_{2}\right)+\alpha_{2} v_{1}+\alpha_{1} f_{1}}{2 t_{1}}$ and the allocation is:

$$
n_{2}^{0}=\frac{t_{1}\left(v_{2}-f_{2}\right)+\alpha_{2} v_{1}-\alpha_{1} f_{1}}{2 \Gamma} \text { and } n_{1}^{0}=\frac{v_{1}+\alpha_{1} n_{2}^{0}}{t_{1}} .
$$

The above formulas confirm that the sales on side 2 have decreased compared to the unconstrained case: $n_{2}^{*}>n_{2}^{0}$.

In the constrained case, platform A offers the service for free on side 1. However, it would benefit from relaxing the zero price constraint and 
therefore setting negative subscription fee. Tying is then a way to achieve this goal by implicitly allowing platforms to offer discounts.

\section{$2.2 \quad$ Tying}

Suppose that the platform can sell another good. In what follows, the term "service" refers to the two-sided platform and the term "good" stands for the extra good that the platform can bundle to the service. Our objective is thus to understand what are the implications of strategies involving tying the good to the service.

For conciseness, we assume that only the consumers of side 1 value the good, while other consumers have no interest in it. In other words, there is perfect correlation between the demand for the good and the potential participation of a member in side 1 . All consumers of side 1 have a homogeneous willingness to pay $\theta$ for the good, assumed for the main part of the paper to be larger than or equal to its cost $c .^{5}$ In this case the platform would sell the good unbundled at price $\theta$, making a positive profit. It can then decide to forego some profit to enhance participation to the platform by deploying a bundling strategy. The case $\theta<c$ corresponds to a situation where it is inefficient for the platform to sell the good alone and will be discussed in a separate section on pure bundling.

Because the demand for the good is inelastic, only two types of bundling strategies are relevant. Indeed if one consumer prefers to buy a bundle instead of the service alone, then all consumers do since they all attach the same value $\theta$ to the good sold by the platform. Thus the service is sold exclusively bundled when some bundling occurs. We refer to the case where only the bundle is sold as "pure bundling", and to the case the bundle and the good alone are sold as "tying". For a one-sided market, Whinston (1990) shows that both in the monopoly case and in the duopoly case, tying has no impact on the market equilibrium, while pure bundling reduces market profitability for all platforms (hence a risk of market foreclosure).

Since $\theta \geq c$, it is optimal for the monopoly platform to use a mixed bundling strategy and thus tying. Indeed compared to pure bundling, tying with $p_{u}=\theta$ leads to the same sales of services while generating a positive revenue from customers not joining the platform. For the moment we focus on tying.

Suppose that the bundle is sold at price $\tilde{p}$. As long as this price is non-

\footnotetext{
${ }^{5}$ One alternative interpretation of the model is that the good is sold on a competitive market at price $\theta$. Then the maximal price at which consumers are willing to buy from the platform is $\theta$. The case $\theta=c$ corresponds to a situation where the platform has access to the same technology as competitive suppliers, while $\theta>c$ corresponds to the case where the platform has access to a superior technology.
} 
negative, only side- 1 consumers consider buying the good. For the reason discussed above, we maintain the assumption that $\tilde{p}$ must be non-negative.

Let $p_{u} \leq \theta$ be the price of the good unbundled. The utility of a side 1 consumer buying the bundle is the same as if she was buying the service at price $\tilde{p}-p_{u}$ and the good at the price $p_{u}$. Since the consumers always buy the good, the implicit price of the service in the bundle, denoted $p_{1}$, is equal to $p_{1}=\tilde{p}-p_{u}$. The demand for the bundle is then $n_{1}=D_{1}\left(p_{1}-\alpha_{1} n_{2}\right)$ while the demand for the good alone is $1-n_{1}$.

The profit under tying is then $\left(p_{1}-f_{1}\right) n_{1}+\left(p_{2}-f_{2}\right) n_{2}+\left(p_{u}-c\right)$ where the prices are constrained by $\tilde{p}=p_{1}+p_{u} \geq 0$ and $p_{u} \leq \theta$. Since $n_{1}$ and $n_{2}$ depend only on $p_{1}$ and $p_{2}$, the optimal price for the good is the monopoly price $p_{u}=\theta$, and thus the implicit price is linked to the price of the bundle by $p_{1}=\tilde{p}-\theta$. The maximal profit is then obtained by solving

$$
\begin{aligned}
\Pi_{T} & =\max _{p_{1}, p_{2}, p_{u}}\left(p_{1}-f_{1}\right) n_{1}+\left(p_{2}-f_{2}\right) n_{2}+(\theta-c) \\
\text { s.t. } & p_{1} \geq-\theta \text { and }(1), p_{u}=\theta .
\end{aligned}
$$

Clearly, the platform prefers tying to no bundling. The reason is that tying allows subsidizing the participation to the platform, while avoiding selling the good to non-interested consumers. Indeed, when side 1 consumers have homogeneous preferences for the good, the only effect of tying is relaxing the constraint without bearing any additional costs. Notice that, albeit its effects on the two-sided service, the same is true for society.

The first order conditions write as:

$$
\begin{aligned}
p_{2}-f_{2}+\alpha_{1}\left(n_{1}+\lambda\right) & =-\frac{n_{2}}{D_{2}^{\prime}}, \\
\left(f_{1}-\alpha_{2} n_{2}-p_{1}\right) D_{1}^{\prime} & =n_{1}+\lambda,
\end{aligned}
$$

where $\lambda$ is the multiplier of the constraint $p_{1} \geq-\theta$. The interpretation is the same as before, one more unit on side 2 raises demand on side 1 by $-\alpha_{1} D_{1}^{\prime}$ and the marginal opportunity cost on side 1 is now $f_{1}-\alpha_{2} n_{2}-p_{1}$.

If the constraint binds, the equilibrium participation levels are thus:

$$
\begin{aligned}
& n_{1}=n_{1}^{0}+\left(1+\frac{\alpha_{1} \sigma}{2 \Gamma}\right) \frac{\theta}{t_{1}} \\
& n_{2}=n_{2}^{0}+\frac{\sigma}{2 \Gamma} \theta
\end{aligned}
$$

with the condition $\alpha_{2} n_{2}-\theta-f_{1} \geq v_{1}+\alpha_{1} n_{2}+\theta$.

Proposition 1 Suppose that the platform deploys tying. The price of the bundle is $\tilde{p}=\max \left\{p_{1}^{*}+\theta, 0\right\}$. Participation is higher on both sides than under no bundling, maximal at $\left(n_{1}^{*}, n_{2}^{*}\right)$ for $\theta \geq-p_{1}^{*}$. 
Proof. Using $\sigma>0$, which is a direct implication of Assumptions 1 and 2, $n_{2}$ increases with $\theta$. Then $n_{1}$ increases with $\theta$ and $n_{2}$. When the constraint is not binding, the solution coincides with the unconstrained monopoly solution.

When the platform uses tying, for $\theta$ small the efficacy of the strategy is also small and the new price is still at a zero level. The implicit subsidy is equal to the value of the tied good and $p_{1}=-\theta$. However, when the value $\theta$ is above the optimal subsidy that an unconstrained platform would set for side 1 , the price of the bundle is positive and reflects any increase in $\theta$.

\subsection{Profits and welfare}

Tying raises profits by relaxing the non-negativity constraint on the price of side 1 and the profit gain is increasing with $\theta$ up to $\theta=-p_{1}^{*}$, constant for $\theta$ above $-p_{1}^{*}$.

We now want to compare the impact on the total social welfare of the decision of the platform. The consumer surplus writes as

$$
C S=\int_{D_{1}^{-1}\left(n_{1}\right)}^{v_{1}} D_{1}(x) d x+\int_{D_{2}^{-1}\left(n_{2}\right)}^{v_{2}} D_{2}(y) d y,
$$

where, $n_{1}$ and $n_{2}$ are the participation levels induced by the monopoly pricing strategy, and $D_{1}^{-1}\left(n_{1}\right)=p_{1}-\alpha_{1} n_{2}$ and $D_{2}^{-1}\left(n_{2}\right)=p_{2}-\alpha_{2} n_{1}$ are the "hedonic" prices on each sides. Given that the good is priced at the monopoly level, consumers obtain no surplus from the market of the good and $C S$ is the consumer surplus under all scenarios. From what precedes, tying a good with positive social value raises participation on both sides.

Proposition 2 Consumer surplus is higher on both sides under tying than under no bundling.

Proof. From equations 4 participation increases with tying. But consumer surplus raises when equilibrium participation levels increase.

The total impact on the welfare is thus unambiguously positive. Notice that due to the homogeneity assumption (inelastic demand for the good), the reduction in sales on the good market doesn't causes any additional welfare losses.

\section{The duopoly case}

To extend the analysis to the duopolistic case, we again base the analysis on the model developed by Armstrong (2005) and Armstrong and Wright (2004). Consumers of each side are located on the unit line and $x$ follows a uniform distribution on each side. There are two platforms, $A$ and $B$, located at the two extremes of the unit interval, $x_{A}=0, x_{B}=1$. They 
compete for agents participation and agents can join only one platform they single-home. If an agent on side $i$ located at $x \in[0,1]$ joins platform $j=A, B$ he enjoys a utility:

$$
u_{i}^{j}=v_{i}-t_{i}\left|x-x_{j}\right|+\alpha_{i} n_{-i}^{j}-p_{i}^{j},
$$

where $p_{i}^{j}$ is the subscription fee, $v_{i}$ is the intrinsic valuation assumed to be platform independent, and $n_{-i}^{j}$ is the mass of consumers on the other side joining platform $j$.

We assume that both platforms are active on both sides and that the market is covered: $0<n_{i}^{B}=1-n_{i}^{A}<1$.

The implied demands of platform $A$ on each side are:

$$
\begin{aligned}
& n_{1}^{A}=\frac{1}{2}+\frac{\alpha_{1}\left(p_{2}^{B}-p_{2}^{A}\right)+t_{2}\left(p_{1}^{B}-p_{1}^{A}\right)}{2 \Gamma} \\
& n_{2}^{A}=\frac{1}{2}+\frac{\alpha_{2}\left(p_{1}^{B}-p_{1}^{A}\right)+t_{1}\left(p_{2}^{B}-p_{2}^{A}\right)}{2 \Gamma} .
\end{aligned}
$$

Note that Assumption 1 guarantees regular demands and concavity of the platforms' problems.

The equilibrium with no constraint on prices is characterized in Armstrong (2005). Symmetric equilibrium unconstrained prices are $p_{1}^{U C}=$ $f_{1}+t_{1}-\alpha_{2}$ and $p_{2}^{U C}=f_{2}+t_{2}-\alpha_{1}$, and the profit of each platform is $\Pi^{U C}=\frac{1}{2}\left(t_{1}-\alpha_{2}+t_{2}-\alpha_{1}\right)$. Assumption 1 implies that this is positive. The consumer surplus with no bundle can be computed to be $S^{U C}=$ $2 v-f_{1}-f_{2}+\frac{3}{2}\left(\alpha_{1}+\alpha_{2}\right)-\frac{5}{4}\left(t_{1}+t_{2}\right)$.

As in the monopolistic case, we want to study how platforms can circumvent equilibrium negative prices by means of tying or pure bundling. We then constraint the prices on side 1 to be non-negative and replace Assumption 2 by the similar condition for a duopoly.

Assumption 2bis $p_{1}^{U C}=f_{1}+t_{1}-\alpha_{2}<0$.

Note that this implies that $p_{2}^{U C}>f_{2}+f_{1}$ since profits are positive. Under Assumption 2bis, platforms set a zero price on side 1 and adjust the prices consequently on side 2 . We show in appendix that constrained symmetric equilibrium prices are:

$$
p_{1}^{C}=0 \text { and } p_{2}^{C}=p_{2}^{U C}+\frac{\alpha_{1}}{t_{1}} p_{1}^{U C}
$$

When the externality $\alpha_{2}$ is large, agents of side 2 attach a high value to the participation of agents of side 1 and therefore platforms subsidize side 1 participation. Unconstrained platforms would set in equilibrium negative prices on side 1 . In this case, the effect on the platform's profit would 
be a loss on side 1 equal to $\frac{t_{1}-\alpha_{2}}{2}<0$ and a gain on side 2 equal to $\frac{t_{2}-\alpha_{1}}{2}$. When the platform is constrained to non-negative pricing, the non-negativity constraint implies a smaller loss equal to $\frac{f_{1}}{2}$ on side 1 and a smaller gain on side 2 .

In the symmetric constrained equilibrium, the two platforms share the market equally and make the following equal profit:

$$
\Pi^{C}=\Pi^{U C}+\frac{\alpha_{1}-t_{1}}{2 t_{1}} p_{1}^{U C} .
$$

which can be verified to be non-negative. The total consumer surplus is

$$
S^{C}=S^{U C}-\frac{\alpha_{1}-t_{1}}{t_{1}} p_{1}^{U C}
$$

The non-negativity price constraint can be beneficial or harmful for consumers depending on the sign of $\alpha_{1}-t_{1}$. The total welfare is unchanged compared to the case where the non-negativity constraint doesn't bind: market shares remain unchanged and platforms readjust prices without welfare losses. Thus the impact on profits and the impact on consumers have opposite signs. One can see that the platforms' equilibrium profit is lower than the unconstrained one if $\alpha_{1}>t_{1}$, higher if $t_{1}>\alpha_{1}$. The reverse holds for consumers surplus.

Typically the constraint raises the prices on one side and reduces them on the other side. Profit decreases if the demand on side 1 is relatively more sensitive to side 2 participation than to an increase in the price of side 1 $\left(\alpha_{1} / t_{1}\right.$ large).

\subsection{Tying}

Suppose now that platform $A$ sells a good of value $\theta$ for side 1 consumers and ties this good to the side 1 service. The stand-alone price of the good is $\theta$ and as in the monopoly case all side 1 consumers buy the good unbundled if they do not join the platform.

The price of the bundle is $\widetilde{p}$, and $p_{1}^{A}=\widetilde{p}-\theta$ is the implicit subscription price in the bundle. Again tying just relaxes the non-negativity price and the platform's $A$ problem becomes:

$$
\begin{aligned}
& \quad \max _{p_{1}^{A}, p_{2}^{A}}\left(p_{1}^{A}-f_{1}\right) n_{1}^{A}+\left(p_{2}^{A}-f_{2}\right) n_{2}^{A}+(\theta-c) \\
& \text { s.t. } p_{1}^{A} \geq-\theta \text { and }(5) .
\end{aligned}
$$

Platform's $B$ problem is unchanged.

The situation is similar to the monopoly case in that for low values of $\theta$ the platform sells the bundle at $\widetilde{p}=0$, while for large values of $\theta$, the nonnegativity constraint is not binding and the allocation becomes independent 
of $\theta$. The critical level of $\theta$ corresponds to the price that platform $A$ would set at the equilibrium of a game where only platforms $B$ is constrained to set a non-negative price on side 1 . Due to the strategic interaction this price differs from $p_{1}^{U C}$ and is computed in appendix to be $-\theta^{A}$, where

$$
\theta^{A}=-\left(\frac{3 \Gamma}{\Delta+2 \Gamma}\right) p_{1}^{U C} .
$$

We thus obtain:

Proposition 3 Under tying, platform $A$ sets a price for the bundle $\tilde{p}=$ $p_{1}^{A}+\theta$, where $p_{1}^{A}=\max \left\{-\theta,-\theta^{A}\right\}$. Platform $B$ sets a zero price on side 1 .

Proof. See Appendix.

When the value of the good is low, platform $A$ always gives the bundle for free. The negative subscription fee is compensated by the value of the bundled product. If Platform $A$ disposes of a good that has high value $(\theta$ big enough), the price of the bundle is positive since the value of the bundled good is bigger than the optimal subsidy.

On side 2, platforms optimally set their prices according to the following response functions:

$$
\begin{aligned}
p_{2}^{A}\left(p_{2}^{B}\right) & =\frac{1}{2} p_{2}^{B}+\frac{1}{2} p_{2}^{C}-\frac{\alpha_{1}+\alpha_{2}}{2 t_{1}} p_{1}^{A} \\
p_{2}^{B}\left(p_{2}^{A}\right) & =\frac{1}{2} p_{2}^{A}+\frac{1}{2} p_{2}^{C}+\frac{\alpha_{2}}{2 t_{1}} p_{1}^{A}
\end{aligned}
$$

where $p_{1}^{A}$ is the equilibrium implicit registration price to platform $A$.

The two best response functions show the standard complementarity between the prices of platform $A$ and $B$ in side 2. However, platforms adjust the price in side 2 by taking into account the discount on the good offered by platform $A$ to participants on side 1 . There are two effects associated with the fact that the implicit subscription fee $p_{1}^{A}$ is now negative.

Demand shifting: demand on side 1 shifts toward platform $A$. On side 2 platform $A$ becomes more attractive. The best reply of platform $B$ shifts downward while the best reply of platform 2 shifts upward.

Competition softening: platform $A$ incurs in higher losses per customer on side 1 and it has less to gain by cutting prices on side 2 and raising demand. As a consequence, platform $A$ tends to set an higher price which shifts its best reply further upwards.

While the demand shifting effect is clearly detrimental to platform's $B$ profit, the competition softening effect on the contrary is beneficial. The 
combination of these two effects determines the optimal pricing strategy of the platforms in side 2 . The equilibrium prices on side 2 are:

$$
\begin{aligned}
& p_{2}^{A}=p_{2}^{C}-\frac{3 \alpha_{1}+\sigma}{3 t_{1}} p_{1}^{A} \\
& p_{2}^{B}=p_{2}^{C}+\frac{\sigma}{3 t_{1}} p_{1}^{A} .
\end{aligned}
$$

An increase in absolute term of the subscription fee $p_{1}^{A}$ results in a higher price $p_{2}^{A}$. Platform $A$ subsides side 1 and recoups on side 2. Depending on the relative magnitude of externalities, the equilibrium reaction of platform $B$ in side 2 may be to decrease or increase its price. If $\sigma>0$ (see Figure 1 left-hand side graph) the "competition softening" strategic effect is not strong enough to offset the effect of the shift in demand and platform $B$ reduces its price when platform $A$ bundles. On the contrary (see Figure 1 right-hand side graph), when the externality is higher on side $1, \sigma<0$, the "competition softening" effect dominates and all prices increase on side 2.
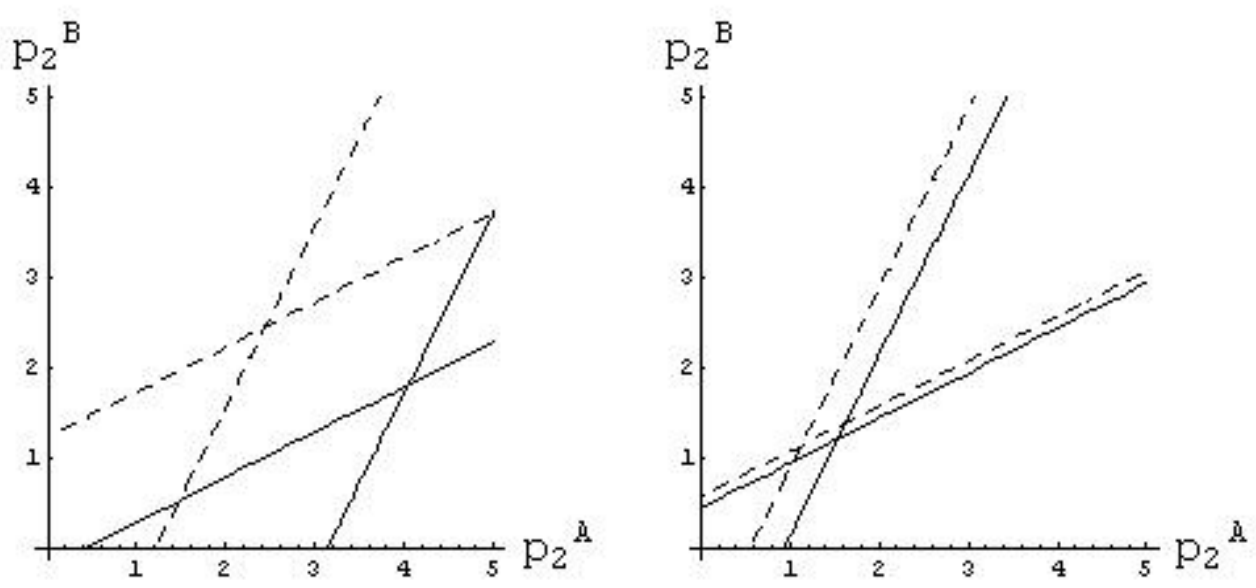

Figure 1: Platforms' best response functions.

However, platform $B$ always sets a lower price than platform $A$ on market 2. The equilibrium platform market shares are then asymmetric given by:

$$
\begin{aligned}
n_{1}^{A} & =\frac{1}{2}-\left(\frac{3 \Gamma+\alpha_{1} \sigma}{6 \Gamma t_{1}}\right) p_{1}^{A} \\
n_{2}^{A} & =\frac{1}{2}-\left(\frac{\sigma}{6 \Gamma}\right) p_{1}^{A}
\end{aligned}
$$


The striking feature is that despite the subsidy of platform $A$ on side 1 , its market share may not increase on side 2 . Indeed its market share on side 2 decreases with tying under circumstances leading the competitor to increase its price, i.e. when the "competition softening" effect dominates $(\sigma<0)$. The reason is that platform $A$ opts for a higher mark up but lower sales on side 2 at given prices of the competitor. This rather counterintuitive results is explained by the fact that the margin is negative on side 1.

An even more counter-intuitive result is that platform $A$ may sell less on side 1 . This occurs when $\alpha_{1}$ is within the top range of admissible values so that $3 \Gamma+\alpha_{1} \sigma<0$.

\subsection{Profits and welfare with tying}

As pointed above, due to the strategic interaction and competition softening effects the impact of tying on the platforms' profit is not obvious. Using the expression $p_{1}^{A}=\max \left\{-\theta,-\theta^{A}\right\}$, total profits are the following:

$$
\begin{aligned}
\Pi_{T}^{A} & =\Phi\left(p_{1}^{A}, 0\right)+(\theta-c) \\
\Pi_{T}^{B} & =\Phi\left(0, p_{1}^{A}\right)
\end{aligned}
$$

where $\Phi(x, y)$ is the profit of platform $i$ in a game where the price of side 1 are fixed to $p_{1}^{i}=x$ and $p_{1}^{j}=y$, and the two platforms compete in price on side 2 . One can show that this profit is

$$
\begin{aligned}
\Phi(x, y)= & \Pi^{C}-\frac{9 \Gamma-\sigma^{2}}{18 t_{1} \Gamma} x^{2}+\frac{\sigma^{2}}{18 t_{1} \Gamma} y^{2} \\
& +\frac{\sigma+3 p_{1}^{U C}}{6 t_{1}} x+\frac{2 \sigma-3 f_{1}}{6 t_{1}} y+\frac{9 \Gamma-2 \sigma^{2}}{18 t_{1} \Gamma} x y .
\end{aligned}
$$

The effect on the profit depends on the relative levels of externalities on each side of the market $\sigma$, and in particular on the equilibrium reaction of the competitor.

Proposition 4 Platform's A profit is higher under tying than under no bundling if and only if the two-sided network externalities asymmetry $\sigma$ is such that $\sigma \leq \frac{(3 \Delta+6 \Gamma) \theta^{A}-(\Delta+5 \Gamma) \min \left\{\theta, \theta^{A}\right\}}{3 \Gamma}$.

Proof. The function $\Pi_{T}^{A}-\left(\Pi^{C}+(\theta-c)\right)$ has the same sign as

$$
-\sigma-3 p_{1}^{U C}-3\left(t_{1}+f_{1}\right)+\frac{\Delta+5 \Gamma}{3 \Gamma} \max \left\{-\theta,-\theta^{A}\right\}>0
$$

which gives the condition. 
The condition always holds if $\sigma<\frac{2 \Delta+\Gamma}{3 \Gamma} \theta^{A}$. Platform $A$ obtains more profits with tying when there are large incentives to subsidize $\left(\theta^{A}\right.$ large) or when the externality on side 1 is not too small compared to side 2 . Notice that $\sigma$ is a measure of the intensity of the price reduction by platform $B$ on side 2. Thus platform's $A$ benefits from tying if platform $B$ does not reduce its price too much, and in particular when it raises its price.

Notice that even if platform $A$ earns a lower profit with tying, tying may occur in equilibrium. Indeed, tying strategies are always optimal ex-post, once the competing platform has set its prices. The platform $A$ may wish to commit ex-ante not to use such a strategy but may lack the credibility to do so.

Therefore there are instances in which platform $A$ may deploy a tying strategy detrimental to its profits.

Proposition 5 Platform's $B$ profit is higher under tying than under no bundling if and only if $\sigma \leq \frac{3}{2} f_{1}+\frac{\sigma^{2}}{6 \Gamma} \min \left\{\theta, \theta^{A}\right\}$.

Proof. $\Pi_{T}^{B}>\Pi^{C}$ if

$$
-2 \sigma+3 f_{1}-\frac{\sigma^{2}}{3 \Gamma} \max \left\{\theta, \theta^{A}\right\}>0
$$

which gives the conditions.

Notice that the condition is met for all $\theta$ if $\sigma$ is small enough. When side 1 benefits more from externalities than side 2 , or slightly less, both platforms benefit from tying. On the contrary, if the externalities are much higher on side 2 both platforms earn lower profits when platform's $A$ undertake a tying strategy. In the middle range, there may be conflicting effect as platform $A$ gains for low value of the bundle while platform $B$ gains for high values.

A striking feature is that for $\sigma>0$ and an implicit subsidy $\min \left\{\theta, \theta^{A}\right\}$ large enough, platform's $B$ profit is higher and platform's $A$ profit is lower when $A$ uses tying. Thus when the market is conducive to high subsidies and platform $A$ can bundle access with a high value good, it would be optimal for platform $A$ to commit not to tie.

A second important consequence is that tying by its competitor cannot hurt a platform unless the subsidy is small and is offered to the low externality side of the market. In particular, if $\sigma \leq \frac{3}{2} f_{1}$, then platform's $B$ profit is larger when platform $A$ uses a tying strategy.

In the following, the impact of tying on the aggregate consumer surplus is analyzed. Because consumers are affected depending on their different locations, distributional effects between sides and locations are also tackled. Focusing on the total consumers surplus we find that the effect is positive when the externality perceived by consumers in side 1 is small. 
Proposition 6 The consumer surplus is always higher on side 1 with tying than with no bundle. It is also higher on side 2 if and only if $\sigma^{2} \min \left\{\theta, \theta^{A}\right\} \geq$ $\frac{18 \alpha_{1} \Gamma^{2}}{t_{1} t_{2}}$.

Proof. The consumer surplus on side $i$ writes as:

$$
S_{i}=v+\alpha_{i} n_{-i}^{B}-t_{i} n_{i}^{B}-p_{i}^{B}+t_{i} \frac{\left(n_{i}^{A}\right)^{2}+\left(n_{i}^{B}\right)^{2}}{2}
$$

The total change in consumer surplus on side 1 is:

$$
S_{1}^{T}-S_{1}^{C}=\left(-p_{1}^{A}\right)\left(\frac{1}{2}+\frac{1}{t_{1}}\left(\frac{3 \Gamma+\alpha_{1} \sigma}{6 \Gamma}\right)^{2}\left(-p_{1}^{A}\right)\right)
$$

On side 2 we obtain the change in surplus:

$$
S_{2}^{T}-S_{2}^{C}=\left(-p_{1}^{A}\right)\left(-\frac{\alpha_{1}}{2 t_{1}}+t_{2}\left(\frac{\sigma}{6 \Gamma}\right)^{2}\left(-p_{1}^{A}\right)\right) .
$$

On side 1 , tying is equivalent to a reduction of prices which benefits consumers. On side 2 , the effect is more ambiguous. The demand shift on side 1 raises the perceived quality of platform $A$ but reduces it on platform $B$. The overall impact is thus ambiguous. Moreover the competition softening effect implies higher prices. In the limit case where $\alpha_{1}=0$, the consumer surplus is higher on both sides. Indeed the utility obtained by the marginal consumer on side 2 (indifferent between $A$ and $B$ ) is independent of the subsidy: the reduction in platform's $B$ price is just enough to maintain at a constant level the utility of the marginal consumer (who shifts to the right) implying that the surplus increases on this side.

Consumers are also affected in a different way depending on their locations. One way to look at this issue is to measure the change in gross utility offered by each platform on each side: $\Delta u_{i}^{l}=\alpha_{i} n_{-i}^{l}-p_{i}^{l}-\left(\alpha_{1} \frac{1}{2}-p_{i}^{C}\right)$.

Proposition 7 When $\alpha_{1}$ is small, platform A proposes a higher utility than platform $B$ to both sides. The reverse is true if $\alpha_{1}$ is large enough (and in particular when $\sigma<0$ ).

Proof. See Appendix.

Thus, provided that the externality on side 1 is not too large, the clients of platform $B$ loose with the introduction of the bundle, while those of platform $A$ benefit from it.

For large values of $\alpha_{1}$, we see again that some counter-intuitive effects may arise. Figure 2 plots these changes in utility. The example sets $t_{1}=1$, $t_{2}=10, \alpha_{1} \alpha_{2}=9$ and $p_{1}^{A}=-1$. Dashed lines depicts utilities of side 1 


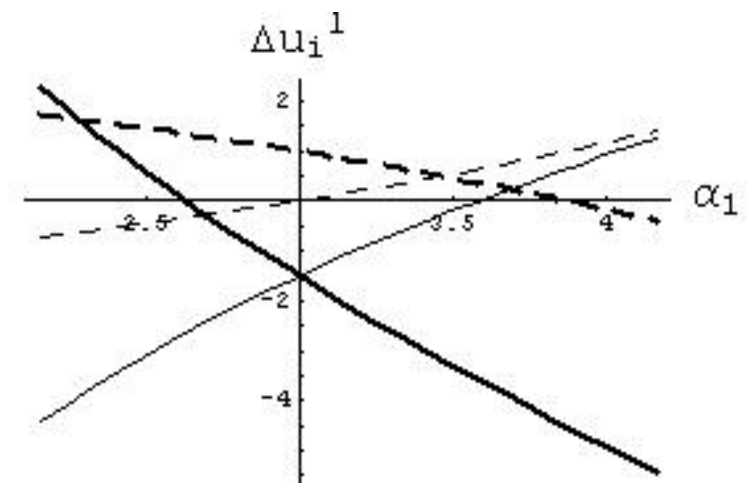

Figure 2: Consumers' utilities.

while the solid lines the ones of side 2 . The thick lines represent the utilities enjoyed by consumers of platform $A$.

The points where curves of the same side cross correspond to equal market shares on that side. For low $\alpha_{2}$ and thus high $\alpha_{1}$, platform $B$ sells more on both sides and customers of platform $A$ receive a lower utility when this platform uses bundles.

Note that there is no instance where all customers benefit from bundling nor instances where they all suffer as a result of bundling. Thus there will always be some conflicts between consumers on the issue of bundling.

Concerning total consumer welfare the effect of tying depends on the degree of asymmetry. The total consumer surplus changes by

$$
S T^{T}-S T^{C}=\left(-\frac{p_{1}^{A}}{2 t_{1}}\right)\left(\frac{\left(3 \Gamma+\alpha_{1} \sigma\right)^{2}+t_{1} t_{1} \sigma^{2}}{18 \Gamma^{2}}\left(-p_{1}^{A}\right)+t_{1}-\alpha_{1}\right)
$$

If $\alpha_{1} \leq t_{1}$ total consumer surplus is higher with tying than with no bundle. Otherwise it is higher if $\sigma$ is large or the subsidy is large.

Total welfare changes according to the following expression:

$$
W^{T}-W^{C}=\left(p_{1}^{A}\right)^{2} \frac{Z}{36 \Gamma^{2} t_{1}}
$$

where $Z=-9 \Gamma^{2}+\sigma\left(6 \alpha_{1}+5 \sigma\right) \Gamma+\alpha_{1} \sigma^{2}\left(2 \alpha_{1}+\sigma\right)$. As we can see, the effect is ambiguous. There can be instances where total welfare increases or decreases. In particular, the zones where total welfare increases are associated with high asymmetry in the membership externality.

\subsubsection{Symmetric two-sided network externalities $\left(\alpha_{1}=\alpha_{2}\right)$}

Assume that the externality is the same on both sides $\sigma=0$ and set $\alpha_{1}=$ $\alpha_{2}=\alpha$. Note that the assumption of unconstrained negative equilibrium 
prices on side 1 and regularity conditions imply that $t_{1}<\alpha<t_{2}$. In words, the market configuration is such that side 1 is more competitive than side 2 and the network effect cannot be too small nor too big with respect the degree of competition.

Platform's $A$ optimal implicit price is now $p_{1}^{A}=\max \left\{-\theta, \frac{1}{2} p_{1}^{U C}\right\}$. Symmetry implies that platform $A$ expands its market share in side 1: $n_{1}^{A}=\frac{1}{2}-$ $\frac{p_{1}^{A}}{2 t_{1}}$. In side 2 , platform $A$, consistently with the general framework, charges higher prices $p_{2}^{A}=p_{2}^{C}-\frac{\alpha}{t_{1}} p_{1}^{A}$. However, due to the symmetry, market shares are left unchanged, $n_{2}^{A}=n_{2}^{B}=\frac{1}{2}$ and platform $B$ maintains also its price unchanged: $p_{2}^{B}=p_{2}^{C}$. In side 2 , symmetry leaves platform $B$ neutral with respect the tying strategy which means that from the point of view of platform $B$ the effects of demand shifting and competition softening cancel out.

Platform $A$ finds always profitable to tie due to higher margins in side 2 which completely offsets the loss in side 1 . Also platform $B$ always benefits. The reduction of losses in side 1 due to the reduction of side 1 market share and neutrality in side 2 allows platform $B$ to enhance its profit with respect the constrained case.

However, the effect on total consumer surplus and total welfare are negative.

Corollary 1 Assume $\sigma=0$. If platform A uses tying, then profit increases for both platforms, total consumer surplus decreases and total welfare decreases.

Proof. $W^{T}-W^{C}$ has the same sign as $-9 \Gamma^{2}<0$. Therefore, total consumer surplus decreases.

Thus tying hurts consumers on side 2 in a significant manner, sufficient to offset the other effects. To understand the result on welfare, notice that when $\sigma=0$, the effect of bundling is only to shift demand toward platform $A$ on side 1 , while the two platforms continue to share the market equally on side 2. However to raise the total value of network effect it is necessary to shift demand on both sides in the same direction. Here the value of network externalities is unchanged because what is gained on side 2 by the customers of platform $A$ is lost by the customers of platform $B$. Thus the only welfare effect is an increase of the total transport cost.

\subsubsection{Network effects only on side $2\left(\alpha_{1}=0\right)$}

Suppose that only side 2 cares about the participation of the other side: $\alpha_{1}=0$. The degree of symmetry $\sigma$ is therefore always positive and equal to $\alpha_{2}$.

The equilibrium prices in side 1 is again $p_{1}^{A}=\max \left\{-\theta, \frac{1}{2} p_{1}^{U C}\right\}$. As in the general case, by subsiding side 1 , platform $A$ consequently charges 
an higher price on side 2 to recoup the losses on side 1 . However in this context, by tying, platform $A$ increases its market shares unambiguously on both sides and platform $B$ reacts by setting lower prices on side 2 . The fact that consumers on side 1 do not benefit from participation eliminates the competition softening effect in favor of the demand shifting effect. Platform $A$ expands the markets share on side 1 due to subsidies. It becomes therefore more attractive on side 2 and it can charge higher prices while expanding its marker shares also in side 2. Tying can be profitable for both platforms when the network effect $\alpha_{2}$ is small. In this case, platform $A$ offsets its losses on side 1 by charging higher margins and expanding its market share in side 2. Platform $B$ then reduces its price on side 2 as a reaction to the shift in demand.

Corollary 2 Assume $\alpha_{1}=0$. If platform A uses tying, then consumer surplus increases on each side. Platform's A profit and total welfare increases if $\alpha_{2}$ is large, while platform's $B$ profit increases and total welfare decreases when $\alpha_{2}$ is small.

Proof. Platform's $A$ profit is larger with tying iff

$$
\alpha_{2}>\min \left\{3\left(f_{1}+t_{1}\right), \frac{3}{2}\left(f_{1}+t_{1}+\theta\right)\right\} .
$$

Platform's $B$ profit is larger iff min $\left\{\theta,-\frac{1}{2}\left(f_{1}+t_{1}-\alpha_{2}\right)\right\}>\frac{6 \Gamma}{\left(\alpha_{2}\right)^{2}}\left(\alpha_{2}-\frac{3}{2} f_{1}\right)$, in particular if $\alpha_{2}<\frac{3}{2} f_{1}$. From consumer surplus increases. $W^{T}-W^{C}$ has the sign of $-9 \Gamma+5 \sigma^{2}$.

Tying is actually beneficial for consumers when there is no network externality on side 1 . Consumers on side 1 benefit from the subsidy and consumers on side 2 on average pays lower prices due to platform $B$ pricing strategy.

\section{Pure bundling vs tying}

While tying is ex-post optimal compared to pure bundling, it could be in the interest of platform $A$ to commit to pure bundling. The motivation for such commitment may differ depending on whether platform $A$ just maximizes its duopoly profit, or the strategy is used to deter platform $B$ from entering the market. In the last case, platform $A$ may choose to minimize platform's $B$ duopoly profit. In this section, we compare the profits under tying and pure bundling.

Suppose the platform sells only a bundle at price $\tilde{p}$. Buying the bundle is equivalent to buying the service at price $\tilde{p}-\theta$ and the good at price $\theta$. Thus we define as before the implicit price of the service in the bundle as 
$p_{1}^{A}=\tilde{p}-\theta$. Under pure bundling, behaviour of platform $B$ is the same as under tying, but the platform's $A$ problem becomes:

$$
\begin{aligned}
& \max _{p_{1}^{A}, p_{2}^{A}}\left(p_{1}^{A}-f_{1}+m\right) n_{1}^{A}+\left(p_{2}^{A}-f_{2}\right) n_{2}^{A} \\
\text { s.t. } & p_{1}^{A} \geq-\theta \text { and }(5)
\end{aligned}
$$

where $m=\theta-c$ is the implicit margin on each unit of good sold.

With pure bundling any additional clients on side 1 generates an additional revenue $m$. As pointed by Whinston (1990), the implications for pricing strategies are equivalent to a reduction in the marginal production cost $f_{1}$ of platform $A$ on side 1 by an amount $m$ in the tying model. Thus the platform prices are the prices that would set a platform using a mixed bundle with a marginal cost $f_{1}-m$.

We provide a full characterization of the equilibrium in appendix. Because of the complexity of the effects, we shall present them sequentially.

Notice that when $m=0$, the equilibrium prices and profits are the same under pure and tying. In what follows, we analyze, starting from $m=0$, the impact of increasing $m$ on the prices and profits under pure bundling. We denote $p_{i}^{j}(m)$ the equilibrium price of platform $j$ on side $i$ $\left(p_{1}^{A}(m)=\tilde{p}(m)-\theta\right)$. The equilibrium prices under tying are thus $p_{i}^{j}(0)$.

\subsection{The opportunity cost effect on side 2}

Suppose that $\theta<\theta^{A}$ and that $m$ is not too far apart from zero. By continuity of the equilibrium, the platform $B$ sets a zero price on side 1 , and the platform $A$ sells the bundle at a zero price. The implicit price of the platform 1 service on side 1 is thus $p_{1}^{A}(m)=p_{1}^{A}(0)=-\theta$.

Considering side 2 the behavior of platform $B$ is unchanged, but the price of platform $A$ is affected. Indeed the opportunity cost of selling on side 2 decreases, since the profitability of extra sales induced on side 1 is augmented by $m$. This leads to a smaller best reply on side 2 of $A$ and equilibrium prices on this side are:

$$
\begin{aligned}
& p_{2}^{A}(m)=p_{2}^{A}(0)-\frac{2 \alpha_{1}}{3 t_{1}} m, \\
& p_{2}^{B}(m)=p_{2}^{B}(0)-\frac{\alpha_{1}}{3 t_{1}} m .
\end{aligned}
$$

The effect of pure bundling is thus to reduce prices on side 2. Since it raises the incentives of platform $A$ to sell on side 1, platform $A$ prices in a more competitive manner on side 2 and platform $B$ follows. Consequently, platforms' market shares are:

$$
\begin{aligned}
& n_{1}^{A}(m)=n_{1}^{A}(0)+\frac{\alpha_{1}^{2}}{6 \Gamma t_{1}} m, \\
& n_{2}^{A}(m)=n_{2}^{A}(0)+\frac{\alpha_{1}}{6 \Gamma} m .
\end{aligned}
$$


The pure bundle direct effect increases platform's $A$ market shares on both sides. The conclusion is thus that pure bundling intensify competition on side 2 .

Proposition 8 Assume that $m$ is positive but close to zero and $\theta<\theta^{A}$. If $\sigma \geq 0$, platform A prefers tying to pure bundling. If $\alpha_{1}$ is small enough, then platform $B$ prefers pure bundling to tying.

Proof. See Appendix.

Thus when the externality on the side where bundling occurs is small, platform $A$ would not benefit from committing to pure bundling as this would reduce the duopoly profit and may even increase the incentives of a competitor to enter compared to tying.

\subsection{The opportunity cost effect on side 1}

Suppose now that $\theta>\theta^{A}$ and that $m$ is not too far apart from zero. Platform $B$ sets a zero price on side 1 , and platform $A$ sells the bundle at a positive price. A new effect is that changing the opportunity cost of selling to side 1 customers affects the price of the bundle. It is shown in appendix that the new implicit equilibrium price is:

$$
p_{1}^{A}(m)=p_{1}^{A}(0)-\rho m \text { where } \rho=\frac{3 \Gamma+\alpha_{1} \sigma}{\Delta+2 \Gamma}
$$

Notice that $\rho>0$ if $\sigma>0$ or is not too large. In this case, we find the intuitive conclusion that the price of the bundle decreases $\left(p_{1}^{A}(m) \leq p_{1}^{A}(0)\right)$.

Accounting for this change in prices, the analysis is the same as before. From equations 6 , prices on side 2 are:

$$
\begin{aligned}
& p_{2}^{A}(m)=p_{2}^{A}(0)-\frac{2 \alpha_{1}}{3 t_{1}} m+\frac{2 \alpha_{1}+\alpha_{2}}{3 t_{1}} \rho m \\
& p_{2}^{B}(m)=p_{2}^{B}(0)-\frac{\alpha_{1}}{3 t_{1}} m-\frac{\sigma}{3 t_{1}} \rho m .
\end{aligned}
$$

Consequently, platform's $A$ market shares are:

$$
\begin{aligned}
& n_{1}^{A}(m)=n_{1}^{A}(0)+\frac{\alpha_{1}^{2}}{6 \Gamma t_{1}} m+\left(\frac{3 \Gamma+\alpha_{1} \sigma}{6 \Gamma t_{1}}\right) \rho m, \\
& n_{2}^{A}(m)=n_{2}^{A}(0)+\frac{\alpha_{1}}{6 \Gamma} m+\left(\frac{\sigma}{6 \Gamma}\right) \rho m .
\end{aligned}
$$

The additional effect reduces the aggressiveness of platform $A$ on side 2 , while the reaction of platform $B$ depends on $\sigma$ with a price reduction if it is positive.

We thus obtain: 
Proposition 9 The conclusions of Proposition 8 holds for $\theta>\theta^{A}$ if $\rho \Phi_{1}\left(p_{1}^{A}(0), 0\right) \geq$ $0($ for $A)$ and $\rho \Phi_{2}\left(0, p_{1}^{A}(0)\right) \leq 0($ for $B)$.

Proof. See Appendix.

It is worth relating these conditions to these obtained in the previous section when comparing the tying profit to the situation with no bundling. Notice that $\Phi(x, 0)$ is concave and $\Phi(0, y)$ is convex, which allows to show that:

Corollary 3 Assume $\rho>0$. If platform A prefers no bundling to tying, it also prefers tying to pure bundling.

If platform $B$ prefers tying to no bundling, it also prefers pure bundling to tying.

Proof. By concavity and convexity

$$
\begin{aligned}
\Pi_{T}^{A}-\Pi^{C}-m & =\Phi\left(p_{1}^{A}(0), 0\right)-\Phi(0,0) \geq \Phi_{1}\left(p_{1}^{A}(0), 0\right) p_{1}^{A}(0) \\
\Pi_{T}^{B}-\Pi^{C} & =\Phi\left(0, p_{1}^{A}(0)\right)-\Phi(0,0) \leq \Phi_{2}\left(0, p_{1}^{A}(0)\right) p_{1}^{A}(0)
\end{aligned}
$$

The result then follows from $p_{1}^{A}(0)<0$.

Under these conditions, in particular when the subsidy with bundling is large enough, we see that platform $A$ will have no reason to use pure bundles to deter entry, nor to enhance its duopoly profits.

\subsection{Large impact on opportunity cost}

When $m$ is far apart from zero, new phenomena may arise. In particular the strategic effects may in some cases induce platform $B$ to increase its price. Indeed we show in appendix, that on side 1, the price of the bundle and/or the price of platform $B$ can be positive.

To understand this issue let us first define a notion of strategic complementarity on side 1 . Define $\hat{p}_{1}^{B}\left(p_{1}^{A}, m\right)$ as the equilibrium price of platform $B$ on side 1 in a game where the price of platform $A$ is constrained to be $p_{1}^{A}$ but the price $p_{1}^{B}$ of platform $B$ is not constrained and can be negative. Then it is shown in appendix, equation (8), that

$$
\begin{aligned}
& \frac{\partial \hat{p}_{1}^{B}\left(p_{1}^{A}, m\right)}{\partial p_{1}^{A}}=\frac{\Delta-\Gamma}{\Delta+2 \Gamma}, \\
& \frac{\partial \hat{p}_{1}^{B}\left(p_{1}^{A}, m\right)}{\partial m}=\frac{\alpha_{1} \sigma}{\Delta+2 \Gamma} .
\end{aligned}
$$

The first derivative shows that the prices on side 1 are "strategic complements" only if $3 \Gamma-\sigma^{2}>0$, which holds if network effects are not too high, 
or if the platforms are differentiated enough. ${ }^{6}$ Notice that $\Delta-\Gamma=3 \Gamma-\sigma^{2}$. Thus the configuration $\Delta>\Gamma$ characterizes a situation in which the difference in the network extenalities between the two sides is small.

However if $\Delta<\Gamma$, decreasing the price of platform $A$ on side 1 leads to an increase of the price of platform $B$ on the same side.

The second derivative shows that the effect on platform's $B$ price depends on the relative intensity of the network effect on both sides. If side 2 has the highest externality, $\sigma>0$, increasing the marginal cost of platform $A$ (reducing $m$ ) leads platform $B$ to reduce its prices.

Notice that the same condition gives the impact of tying on the price of platform $B$ on side 2 . In particular, the unconstrained price of $B$ on side 1 decreases with $m$ under circumstances that leads $B$ to increase its price on side 2 when tying is introduced.

One interpretation is to see that the impact of $m$ for a given $p_{1}^{A}$ is that platform $A$ reduces its price $p_{2}^{A}$. Thus the residual demand faced by platform $B$ on side 2 decreases. From the monopoly pricing formula (2), notice that $\frac{\partial p_{1}^{*}}{\partial v_{2}}=\frac{t_{1} \sigma}{\Delta}$. If $\sigma>0$, the platform increases its price on side 1 when its value on side 2 decreases, which is the case for the residual demand when $p_{2}^{A}$ decreases.

In other words, the marginal benefit of raising demand on side 1 increases with the demand of the other side when side 1 is the low externality side $(\sigma>$ $0)$. Then the unconstrained price on side 1 decreases with the demand on side 2. Because pure bundling makes $A$ more aggressive on side 2, platform $B$ is relatively less attractive and its price increases on side 1 .

On the opposite if $\sigma<0$ a platform facing an increase in the demand of side 2 would exploit this opportunity by simply raising its price on side 1 .

Overall we see that when $\sigma>0$, increasing $m$ raises the optimal price of $B$ on side 1 . Then for large values of $m$ two new types of equilibria may arise.

First, all the prices may be positive and thus coincide with the equilibrium allocation of an unconstrained game where the cost of platform $A$ on side 1 is $f_{1}-m$.

Second, In the case where $\Delta>\Gamma$, there may then be an intermediate range of values $\theta$ such that platform $A$ gives the bundle for free while platform $B$ chooses a positive price on side 1 .

Similarly, when $\Delta<\Gamma$, for low values of $\theta$, platform $B$ may choose a positive price and not platform $A$.

The comparison between tying and pure bundling profit becomes then very complex so that we don't pursue it further (the equilibrium allocation are presented in appendix).

\footnotetext{
${ }^{6}$ The explicit condition is $3 t_{1} t_{2}>\alpha_{1}^{2}+\alpha_{2}^{2}+\alpha_{1} \alpha_{2}$.
} 


\subsection{Symmetric network effect $\left(\alpha_{1}=\alpha_{2}\right)$}

In this example we study the case of pure bundling under the hypothesis of symmetric network externalities between the two sides $\left(\alpha_{1}=\alpha_{2}=\alpha\right)$. In this case, given $\sigma=0$, it is immediate to see that $\hat{p}_{1}^{B}\left(p_{1}^{A}, m\right)$ increases with $p_{1}^{A}$ and is independent of $m$. Thus platform $B$ never chooses a positive price on side 1. Platform $A$ then charges

$$
p_{1}^{A}(m)=\max \left\{-\theta,-\theta^{A}-\frac{1}{2} m\right\}
$$

while platform $B$ is still constrained. Under symmetry there are thus only two relevant pricing regimes to study.

Demands are:

$$
\begin{aligned}
& n_{1}^{A}(m)=\frac{1}{2}-\frac{p_{1}^{A}(m)}{2 t_{1}}+\frac{\alpha^{2}}{6 \Gamma t_{1}} m \\
& n_{2}^{A}(m)=\frac{1}{2}+\frac{\alpha}{6 \Gamma} m
\end{aligned}
$$

Platform A sells more under pure bundling than under tying or no bundling on both markets.

We then obtain:

Proposition 10 If $\sigma=0$, platform A prefers tying to pure bundling, and at least for $\alpha$ small platform $B$ prefers pure bundling to tying.

Proof. See Appendix.

Thus, at least for the case of symmetric externalities, pure bundling is unlikely to emerge, irrespective of whether Platform $A$ accomodate or trie to foreclosure the market to $B$.

\section{Bundling inefficient goods: pure bundling vs no bundling}

In what precedes we assumed that $m=\theta-c>0$ which corresponds to the case where the firm has some market power that allows it to sell the good at a price above cost. As already pointed, when such a possibility doesn't exist, the platform may still subsidize participation by bundling a good such that $m<0$ but $\theta>0$ and setting a price below $\theta$ for the bundle. Under such a circumstance, the good will not be sold unbundled as this would involve a loss. ${ }^{7}$ In this section we allow for $m<0$, and extends the analysis to pure bundling. ${ }^{8}$

\footnotetext{
${ }^{7}$ In the case $m<0$, the platform may wish to commit to tying (in duopoly) but ex-post it could always set a price $c$ and not sell. Thus the platform would need to commit to set a price $\theta$ for the good alone along with a tying strategy.

${ }^{8}$ Notice that platform $B$ could follow the same strategy. We ignore this issue here so that the question is whether there is an equilibrium where some platform commits to
} 


\subsection{Monopoly}

As before the bundle is sold at price $\tilde{p}$, and the implicit price of the service is $p_{1}=\tilde{p}-\theta$. In the monopoly case, the demand of bundle on side 1 is then equal to $D_{1}\left(p_{1}-\alpha_{1} n_{2}\right)$, leading to a profit for the monopoly platform:

$$
\begin{aligned}
\Pi_{P} & =\max _{p_{1}, p_{2}}\left(p_{1}-f_{1}\right) n_{1}+\left(p_{2}-f_{2}\right) n_{2}+m . n_{1} \\
\text { s.t. } p_{1} & \geq-\theta
\end{aligned}
$$

where the demand $n_{1}$ and $n_{2}$ are solutions of (1) as before.

As already mentioned the equilibrium prices and the equilibrium allocation are the same as with tying by a platform with a marginal cost $f_{1}-m$ on side 1. Equation 3 and 4 show that the participation levels are decreasing with the marginal cost:

Proposition 11 Consider a monopoly platform selling a pure bundle. Then the participation levels are nondecreasing with $\theta$ and $m$ on both sides.

Proof. If the constraint is binding, the allocation is given by (4), evaluated for a cost $f_{1}-m$ which defines increasing quantities. When the constraint is not binding:

$$
\begin{aligned}
& n_{1}=n_{1}^{*}+\frac{2 t_{2}}{\Delta} m \\
& n_{2}=n_{2}^{*}+\frac{\left(\alpha_{1}+\alpha_{2}\right)}{\Delta} m
\end{aligned}
$$

again increasing with $m$. Thus participation increases with $\theta$.

When $m$ is negative but close to zero, a pure bundle induces participation levels close to the mixed bundle, and thus above the unbundled case. But when $m$ is large negative relative to $\theta$, in particular if the value $\theta$ is small and the cost $c$ is high, participation with a pure bundle will be smaller than with no bundle.

A direct computation shows also that the price of the pure bundle is

$$
\tilde{p}=\max \left\{p_{1}^{*}+\theta-\frac{2 \Gamma+\alpha_{1} \sigma}{\Delta}(\theta-c), 0\right\} .
$$

When externalities are small, the price of the bundle is monotonic with $\theta$. But the case where $2 \Gamma-\alpha_{2} \sigma<0$ is more counter-intuitive as the price of the bundle is non-increasing with $\theta$. In this case the bundle is sold at a zero price for values $\theta$ above some threshold.

In general pure bundling implies a gain (relaxing the no-subsidy constraint) and a loss since the good is sold at a price below cost if $m<0$ and some profitable sales are foregone if $m>0$. It is immediate that the pure

bundling. 
bundling profit is increasing with $\theta$ (as long as the constraint binds) and with the margin $m$.

Given that pure bundling and tying yield the same positive gain in profit for a zero margin, we obtain:

Proposition 12 For any given $\theta$, there exists $m_{\theta}<0$ such that $\Pi_{P}-\Pi^{*}$ is positive for $0 \geq m>m_{\theta}$.

Concerning the impact on consumer surplus we should account for the fact that the objective of the platform bundling is to raise participation which should benefit consumers.

Conjecture 1 If platform $A$ is a monopoly and $\Pi_{P}-\Pi^{*}>0$, then consumer surplus is higher on both sides under pure bundling than under no bundling.

\subsection{Duopoly}

Considering the duopoly case, the analysis is similar to the one developed in the preceding section except that now $m$ can be negative. When $m$ is close to zero, the allocation and the profits with pure bundling will be close to the allocation with tying. The previous conclusions on the comparison between tying and no bundling apply to the comparison between pure bundling and no bundling.

However the comparison between pure and tying is reversed. In particular for $\sigma=0$ and $m<0$, platform $A$ prefers pure bundling to tying.

For $m$ large negative, the analysis parallels the analysis of the previous section (See Appendix), and any prices may become positive on side 1. To simplify matters we present the case of a symmetric network effects.

So assume $\sigma=0$ which implies $\alpha_{1}=\alpha_{2}=\alpha$. Under symmetry there are only three relevant pricing regimes to study. The first case occurs when $m>p_{1}^{U C}+2 \theta$ and both platforms are constrained to set zero prices. In the second one, $3 p_{1}^{U C}<m<p_{1}^{U C}+2 \theta$, platform $A$ charges a positive price $p_{1}^{A}=$ $\frac{1}{2} p_{1}^{U C}-\frac{1}{2} m$, while platform $B$ is still constrained. In the third one which occurs when $m<3 p_{1}^{U C}$, both platforms charge positive prices (respectively, $p_{1}^{A}=p_{1}^{U C}-\frac{2}{3} m$ and $p_{1}^{B}=p_{1}^{U C}-\frac{1}{3} m$ ). Therefore, under symmetry, platform $A$ never suffers from a situation in which she is constrained and the rival is not. Note also that the case when both platforms can charge positive prices arises only when $m<0$.

The impact on the profits' increment with respect the constrained case (and therefore on the incentives to pure bundle) is complex. We illustrate the effects with an example obtained by letting $\theta$ and $\alpha$ varying with $\Gamma=10$, $t_{1}=1, f_{1}=1 / 2$ and $c=1 / 2$. Figure 3 shows the zone of positivity and 
negativity of the profits increments of both platforms compared to no bundle, by plotting the change in profit for values of $\alpha$.

We see that platform $B$ benefits unless $m$ is large positive. Platform $A$ benefits as long as $\theta$ is not too small, thus for $m$ mildly negative. When $m$ is very low, platform $A$ would choose not to bundle the good.

The pattern for the profit gains obtained for $m<0$ is the same for the other regimes: platform $B$ always prefers pure bundle to no bundle, platform $A$ also unless $m$ is very small negative. The difference is that for $m$ large positive the gain of platform $A$ may be negative.
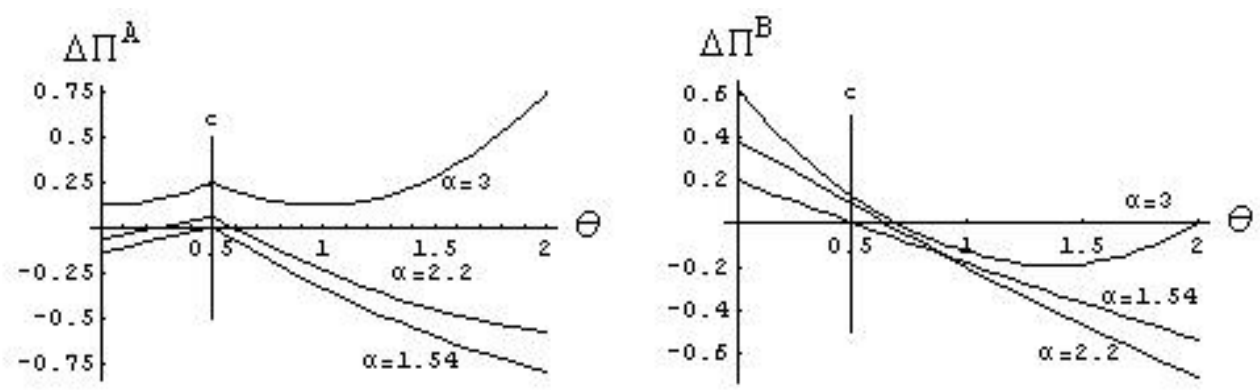

Figure 3: Platforms' profits

Thus low social value of the good determines a situation in which platform $\mathrm{B}$ benefits from bundling (competition softening effect). However, when the value of the good becomes large positive the standard foreclosing effect may arise since platform B can be hurt. This confirms the results that in a two-sided framework there could be foreclosure through bundling.
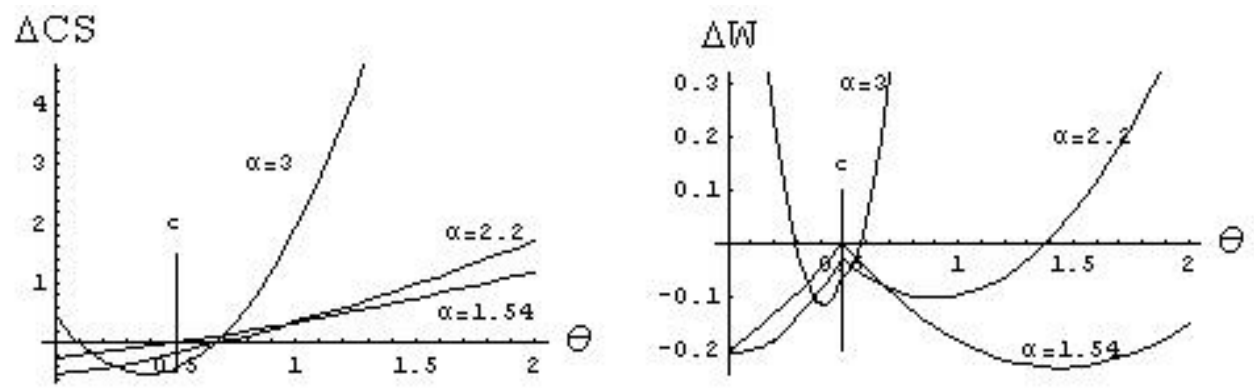

Figure 4: Consumers' surplus and total welfare

Figure 4 depicts the consumer surplus and total welfare effects under symmetry. 
The graph shows that the conclusion that when $\alpha_{1}=\alpha_{2}$, tying has a negative impact on consumer surplus and welfare, extends to the case of pure bundling when $m<0$. The same conclusion applies to the other two regimes.

Notice however that pure bundle can both profitable for $A$ and for welfare if $m$ is positive. 


\section{References}

Adams, W. and J. Yellen (1976). Commodity Bundling and the Burden of Monopoly. The Quarterly Journal of Economics 90(3), 475-498.

Armstrong, M. (2005). Competition in Two-Sided Markets. Rand Journal of Economics forthcoming.

Armstrong, M. and J. Wright (2004). Two-sided markets with multihoming and exclusive dealing.

Caillaud, B. and B. Jullien (2003). Chicken \& Egg: Competition Among Intermediation Service Providers. RAND Journal of Economics 34(2), 309-328.

Choi, J. P. (2004). Antitrust Analysis of Tying Arrangements. SSRN eLibrary.

Evans, D. (2003a). Some Empirical Aspects of Multi-sided Platform Industries. Review of Network Economics 2(3), 191-209.

Evans, D. (2003b). The Antitrust Economics of Two-Sided Markets. Yale Journal of Regulation 20(2), 325-381.

Farhi, E. and A. Hagiu (2004). Tying in Two-Sided Markets: Top Dog, Fat-Cat, Lean and Hungry or Puppy Dog? Working Paper.

Jullien, B. (2001). Competing in Network Industries: Divide and Conquer. IDEI and GREMAQ, University of Toulouse.

Jullien, B. (2005). Two-sided Markets and Electronic Intermediaries. CESifo Economic Studies 51(2-3), 233-260.

Rochet, J. and J. Tirole (2003). An Economic Analysis of the Determination of Interchange Fees in Payment Card Systems. Review of Network Economics 2(2), 69-79.

Rochet, J. and J. Tirole (2005). Two-Sided Markets: A Progress Report. Rand Journal of Economics.

Schmalensee, R. (1984). Gaussian Demand and Commodity Bundling. The Journal of Business 57(1), 211-230.

Whinston, M. (1990). Tying, Foreclosure, and Exclusion. The American Economic Review 80(4), 837-859. 


\section{A The monopoly case}

Proof. For the proofs we may rely on the inverse demand, which can be written as

$$
p_{i}=v_{i}-n_{i} t_{i}+\alpha_{i} n_{-i},
$$

where $v_{i}-n_{i} t_{i}$ is the inverse of the demand function $D_{i}$, and will be referred to as the "hedonic price". The profit function is

$$
\begin{gathered}
\max _{n_{1}, n_{2}}\left(v_{1}-n_{1} t_{1}-f_{1}\right) n_{1}+\left(v_{2}-n_{2} t_{2}-f_{2}\right) n_{2}+\left(\alpha_{1}+\alpha_{2}\right) n_{1} n_{2} \\
\text { s.t. } \quad p_{1}=v_{1}-n_{1} t_{1}+\alpha_{1} n_{2} \geq 0
\end{gathered}
$$

The optimal quantities obtained at

$$
\begin{array}{ll}
v_{1}-n_{1} t_{1}-t_{1} n_{1}+\left(\alpha_{1}+\alpha_{2}\right) n_{2}= & f_{1}+\lambda t_{1} \\
v_{2}-n_{2} t_{2}-t_{2} n_{2}+\left(\alpha_{1}+\alpha_{2}\right) n_{1}= & f_{2}-\lambda \alpha_{1}
\end{array}
$$

where $\lambda$ is non negative and is the multiplier of the non-negativity constraint. The solution reduces to

$$
\begin{aligned}
v_{2}-n_{2} t_{2}-t_{2} n_{2}+\alpha_{2} n_{1}+\left(n_{1}+\lambda\right) \alpha_{1} & =f_{2} \\
\lambda\left(v_{1}-n_{1} t_{1}+\alpha_{1} n_{2}\right) & =0 \\
\frac{\alpha_{2} n_{2}-f_{1}}{t_{1}} & =n_{1}+\lambda \geq n_{1}
\end{aligned}
$$

which gives the first order conditions.

When bundling is used we have to consider the program

$$
\begin{aligned}
\max _{n_{1}, n_{2}} & \left(v_{1}-n_{1} t_{1}-f_{1}+\gamma m\right) n_{1}+\left(v_{2}-n_{2} t_{2}-f_{2}\right) n_{2}+\left(\alpha_{1}+\alpha_{2}\right) n_{1} n_{2}+(1-\gamma) m \\
\text { s.t. } & v_{1}-n_{1} t_{1}+\alpha_{1} n_{2}+\theta \geq 0
\end{aligned}
$$

where $\gamma=1$ if there is pure bundling, $\gamma=0$ if there is mixed bundling. The solution is

$$
\begin{aligned}
v_{1}-n_{1} t_{1}-t_{1} n_{1}+\left(\alpha_{1}+\alpha_{2}\right) n_{2} & =f_{1}-\gamma m+\lambda t_{1} \\
v_{2}-n_{2} t_{2}-t_{2} n_{2}+\left(\alpha_{1}+\alpha_{2}\right) n_{1} & =f_{2}-\lambda \alpha_{1} \\
\lambda\left(v_{1}-n_{1} t_{1}+\alpha_{1} n_{2}+\theta\right) & =0
\end{aligned}
$$

which yield the new first order conditions.

\section{B Duopoly}

\section{B.1 Duopoly with no bundle}

Proof. Denote by $\lambda^{A}$ the multiplier associated to the non-negativity constraint. Taking derivatives of the Lagrangians we obtain the following first 
order conditions for platform $A$ :

$$
\begin{aligned}
& 0=\frac{1}{2}+\frac{\alpha_{1}\left(p_{2}^{B}-p_{2}^{A}\right)+t_{2}\left(p_{1}^{B}-p_{1}^{A}\right)}{2 \Gamma}-\frac{\alpha_{2}\left(p_{2}^{A}-f_{2}\right)}{2 \Gamma}-\frac{t_{2}\left(p_{1}^{A}-f_{1}\right)}{2 \Gamma}+\lambda^{A} \\
& 0=\frac{1}{2}+\frac{\alpha_{2}\left(p_{1}^{B}-p_{1}^{A}\right)+t_{1}\left(p_{2}^{B}-p_{2}^{A}\right)}{2 \Gamma}-\frac{t_{1}\left(p_{2}^{A}-f_{2}\right)}{2 \Gamma}-\frac{\alpha_{1}\left(p_{1}^{A}-f_{1}\right)}{2 \Gamma}
\end{aligned}
$$

and the symmetric for $B$. If $p_{1}^{U C}$ is negative, both the problems of the platforms are constrained. Therefore, setting $p_{1}^{A}=p_{1}^{B}=0$ and $\lambda^{A}=\lambda^{B}>$ 0 , we obtain the following optimal response functions, for platform $A$ :

$$
\begin{aligned}
& p_{2}^{A}\left(p_{2}^{B}\right)=\frac{1}{2} p_{2}^{B}+\frac{f_{2}+t_{2}-\alpha_{1}}{2}+\frac{\alpha_{1}}{2 t_{1}}\left(f_{1}+t_{1}-\alpha_{2}\right) \\
& p_{2}^{B}\left(p_{2}^{A}\right)=\frac{1}{2} p_{2}^{A}+\frac{f_{2}+t_{2}-\alpha_{1}}{2}+\frac{\alpha_{1}}{2 t_{1}}\left(f_{1}+t_{1}-\alpha_{2}\right)
\end{aligned}
$$

The optimal constrained symmetric equilibrium prices are:

$$
\begin{aligned}
& p_{1}^{C}=0 \\
& p_{2}^{C}=f_{2}+t_{2}-\alpha_{1}+\frac{\alpha_{1}}{t_{1}}\left(f_{1}+t_{1}-\alpha_{2}\right)
\end{aligned}
$$

with :

$$
\lambda=-\frac{f_{1}+t_{1}-\alpha_{2}}{2 t_{1}}>0 .
$$

Platforms share the market equally and the equilibrium profits are

$$
\Pi^{C}=\Pi^{U C}+\frac{\alpha_{1}-t_{1}}{2 t_{1}}\left(f_{1}+t_{1}-\alpha_{2}\right)
$$

\section{B.2 Duopoly with mixed bundle}

Proof of Proposition 3. When platform $A$ uses mixed bundles, first order conditions yield

$$
\begin{aligned}
0 & =\frac{1}{2}+\frac{\alpha_{1}\left(p_{2}^{B}-p_{2}^{A}\right)+t_{2}\left(p_{1}^{B}-p_{1}^{A}\right)}{2 \Gamma}-\frac{\alpha_{2}\left(p_{2}^{A}-f_{2}\right)}{2 \Gamma}-\frac{t_{2}\left(p_{1}^{A}-f_{1}\right)}{2 \Gamma}+\lambda^{A}, \\
0 & =\frac{1}{2}-\frac{\alpha_{1}\left(p_{2}^{B}-p_{2}^{A}\right)+t_{2}\left(p_{1}^{B}-p_{1}^{A}\right)}{2 \Gamma}-\frac{\alpha_{2}\left(p_{2}^{B}-f_{2}\right)}{2 \Gamma}-\frac{t_{2}\left(p_{1}^{B}-f_{1}\right)}{2 \Gamma}+\lambda^{B},
\end{aligned}
$$

on side 1 , and

$$
\begin{aligned}
& 0=\frac{1}{2}+\frac{\alpha_{2}\left(p_{1}^{B}-p_{1}^{A}\right)+t_{1}\left(p_{2}^{B}-p_{2}^{A}\right)}{2 \Gamma}-\frac{t_{1}\left(p_{2}^{A}-f_{2}\right)}{2 \Gamma}-\frac{\alpha_{1}\left(p_{1}^{A}-f_{1}\right)}{2 \Gamma} \\
& 0=\frac{1}{2}-\frac{\alpha_{2}\left(p_{1}^{B}-p_{1}^{A}\right)+t_{1}\left(p_{2}^{B}-p_{2}^{A}\right)}{2 \Gamma}-\frac{t_{1}\left(p_{2}^{B}-f_{2}\right)}{2 \Gamma}-\frac{\alpha_{1}\left(p_{1}^{B}-f_{1}\right)}{2 \Gamma}
\end{aligned}
$$


on side 2 .

First, for given prices $p_{1}^{A}$ and $p_{1}^{B}$ on side 1, the optimal response functions on side 2 are:

$$
\begin{aligned}
& p_{2}^{A}\left(p_{2}^{B}\right)=\frac{1}{2} p_{2}^{B}+\frac{1}{2} p_{2}^{C}-\frac{\alpha_{1}+\alpha_{2}}{2 t_{1}} p_{1}^{A}+\frac{\alpha_{2}}{2 t_{1}} p_{1}^{B} \\
& p_{2}^{B}\left(p_{2}^{A}\right)=\frac{1}{2} p_{2}^{A}+\frac{1}{2} p_{2}^{C}-\frac{\alpha_{1}+\alpha_{2}}{2 t_{1}} p_{1}^{B}+\frac{\alpha_{2}}{2 t_{1}} p_{1}^{A} .
\end{aligned}
$$

Solving for these prices we obtain

$p_{2}^{A}=p_{2}^{C}-\left(\alpha_{2}+2 \alpha_{1}\right) \frac{p_{1}^{A}}{3 t_{1}}+\left(\alpha_{2}-\alpha_{1}\right) \frac{p_{1}^{B}}{3 t_{1}}$ and $p_{2}^{B}=p_{2}^{C}-\left(\alpha_{2}+2 \alpha_{1}\right) \frac{p_{1}^{B}}{3 t_{1}}+\left(\alpha_{2}-\alpha_{1}\right) \frac{p_{1}^{A}}{3 t_{1}}$.

The first order conditions on market 1 then write:

$$
\begin{aligned}
\lambda^{A} & =\frac{(\Delta+2 \Gamma) p_{1}^{A}-(\Delta-\Gamma) p_{1}^{B}}{6 \Gamma t_{1}}-\frac{\left(f_{1}+t_{1}-\alpha_{2}\right)}{2 t_{1}} \\
\lambda^{B} & =\frac{(\Delta+2 \Gamma) p_{1}^{B}-(\Delta-\Gamma) p_{1}^{A}}{6 \Gamma t_{1}}-\frac{\left(f_{1}+t_{1}-\alpha_{2}\right)}{2 t_{1}}
\end{aligned}
$$

First notice that it is not possible that no constraint binds.

Now suppose that both constraints are still binding. Therefore the prices are $p_{1}^{A}=-\theta$ and $p_{1}^{B}=0$. Observe that $\lambda^{A}<\lambda^{B}$ which implies that this is the solution if $\lambda^{A}>0$ or $p_{1}^{A}=-\theta>-\theta^{A}=\left(f_{1}+t_{1}-\alpha_{2}\right) \frac{3 \Gamma}{\Delta+2 \Gamma}$.

Now suppose $\theta>\theta^{A}$. Setting $\lambda^{A}=0$ and $\lambda^{B}>0$, the equilibrium prices are $p_{1}^{A}=-\theta^{A}$ and $p_{1}^{B}=0$.we verify that:

$$
\lambda^{B}=-\frac{2 \Delta+\Gamma}{2 t_{1}(\Delta+2 \Gamma)}\left(f_{1}+t_{1}-\alpha_{2}\right)>0 .
$$

The prices on side 2 are then

$$
\begin{aligned}
& p_{2}^{A}=p_{2}^{C}-\left(\alpha_{2}+2 \alpha_{1}\right) \frac{\left(-\theta^{A}\right)}{3 t_{1}} \\
& p_{2}^{B}=p_{2}^{C}+\left(\alpha_{2}-\alpha_{1}\right) \frac{\left(-\theta^{A}\right)}{3 t_{1}}
\end{aligned}
$$

Proof of proposition 7 .

$$
\begin{aligned}
\alpha_{1} n_{2}^{A}-p_{1}^{A}-\alpha_{1} \frac{1}{2} & =\left(\frac{\alpha_{1}\left(\alpha_{2}-\alpha_{1}\right)+6 \Gamma}{6 \Gamma}\right)\left(-p_{1}^{A}\right) \\
\alpha_{2} n_{1}^{A}-p_{2}^{A}-\left(\alpha_{2} \frac{1}{2}-p_{2}^{C}\right) & =\left(\frac{t_{1} t_{2}\left(\alpha_{2}-\alpha_{1}\right)-3 \alpha_{1} \Gamma}{2 \Gamma}\right)\left(-\frac{p_{1}^{A}}{3 t_{1}}\right) \\
\alpha_{1} n_{2}^{B}-\alpha_{1} \frac{1}{2} & =\alpha_{1}\left(\frac{\alpha_{2}-\alpha_{1}}{6 \Gamma}\right) p_{1}^{A} \\
\alpha_{2} n_{1}^{B}-p_{2}^{B}-\left(\alpha_{2} \frac{1}{2}-p_{2}^{C}\right) & =\left(\frac{t_{1} t_{2}\left(\alpha_{2}-\alpha_{1}\right)+3 \alpha_{1} \Gamma}{2 \Gamma}\right) \frac{p_{1}^{A}}{3 t_{1}}
\end{aligned}
$$


For $\alpha_{1}$ close to zero, the first two are positive and the last two are negative. But the reverse holds if $\alpha_{1}-\alpha_{2}$ is large positive and $\Gamma$ is small.

\section{B.3 Duopoly with pure bundle}

Proof. When platform $A$ sells a pure bundle, its problem is thus:

$$
\begin{aligned}
& \max _{p_{1}^{A}, p_{2}^{A}}\left(p_{1}^{A}-f_{1}+m\right) n_{1}^{A}+\left(p_{2}^{A}-f_{2}\right) n_{2}^{A} \\
\text { s.t. } & p_{1}^{A} \geq-\theta \text { and }(5)
\end{aligned}
$$

while platform's $B$ problem is unchanged. When platform $A$ uses mixed bundles, first order conditions yield

$$
\begin{aligned}
0 & =\frac{1}{2}+\frac{\alpha_{1}\left(p_{2}^{B}-p_{2}^{A}\right)+t_{2}\left(p_{1}^{B}-p_{1}^{A}\right)}{2 \Gamma}-\frac{\alpha_{2}\left(p_{2}^{A}-f_{2}\right)}{2 \Gamma}-\frac{t_{2}\left(p_{1}^{A}-f_{1}+m\right)}{2 \Gamma}+\lambda^{A} \\
0 & =\frac{1}{2}-\frac{\left.\alpha_{1}\left(p_{2}^{B}-p_{2}^{A}\right)+t_{2}\left(p_{1}^{B}-p_{1}^{A}\right)\right)}{2 \Gamma}-\frac{\alpha_{2}\left(p_{2}^{B}-f_{2}\right)}{2 \Gamma}-\frac{t_{2}\left(p_{1}^{B}-f_{1}\right)}{2 \Gamma}+\lambda^{B}
\end{aligned}
$$

on side 1 , and

$$
\begin{aligned}
& 0=\frac{1}{2}+\frac{\alpha_{2}\left(p_{1}^{B}-p_{1}^{A}\right)+t_{1}\left(p_{2}^{B}-p_{2}^{A}\right)}{2 \Gamma}-\frac{t_{1}\left(p_{2}^{A}-f_{2}\right)}{2 \Gamma}-\frac{\alpha_{1}\left(p_{1}^{A}-f_{1}+m\right)}{2 \Gamma} \\
& 0=\frac{1}{2}-\frac{\alpha_{2}\left(p_{1}^{B}-p_{1}^{A}\right)+t_{1}\left(p_{2}^{B}-p_{2}^{A}\right)}{2 \Gamma}-\frac{t_{1}\left(p_{2}^{B}-f_{2}\right)}{2 \Gamma}-\frac{\alpha_{1}\left(p_{1}^{B}-f_{1}\right)}{2 \Gamma}
\end{aligned}
$$

on side 2 .

First, for given prices $p_{1}^{A}$ and $p_{1}^{B}$ on side 1, the optimal response functions on side 2 are:

$$
\begin{aligned}
& p_{2}^{A}\left(p_{2}^{B}\right)=\frac{1}{2} p_{2}^{B}+\frac{1}{2} p_{2}^{C}-\frac{\alpha_{1}}{2 t_{1}} m-\frac{\alpha_{1}+\alpha_{2}}{2 t_{1}} p_{1}^{A}+\frac{\alpha_{2}}{2 t_{1}} p_{1}^{B} \\
& p_{2}^{B}\left(p_{2}^{A}\right)=\frac{1}{2} p_{2}^{A}+\frac{1}{2} p_{2}^{C}-\frac{\alpha_{1}+\alpha_{2}}{2 t_{1}} p_{1}^{B}+\frac{\alpha_{2}}{2 t_{1}} p_{1}^{A} .
\end{aligned}
$$

Solving for these prices we obtain

$$
\begin{aligned}
& p_{2}^{A}=p_{2}^{C}-\frac{2 \alpha_{1}}{3 t_{1}} m-\left(\alpha_{2}+2 \alpha_{1}\right) \frac{p_{1}^{A}}{3 t_{1}}+\left(\alpha_{2}-\alpha_{1}\right) \frac{p_{1}^{B}}{3 t_{1}} \\
& p_{2}^{B}=p_{2}^{C}-\frac{\alpha_{1}}{3 t_{1}} m-\left(\alpha_{2}+2 \alpha_{1}\right) \frac{p_{1}^{B}}{3 t_{1}}+\left(\alpha_{2}-\alpha_{1}\right) \frac{p_{1}^{A}}{3 t_{1}} .
\end{aligned}
$$

The first order conditions on market 1 then write:

$$
\begin{aligned}
\lambda^{A} & =\frac{(\Delta+2 \Gamma) p_{1}^{A}-(\Delta-\Gamma) p_{1}^{B}-3 \Gamma p_{1}^{U C}+\left(3 \Gamma+\alpha_{1}\left(\alpha_{2}-\alpha_{1}\right)\right) m}{6 \Gamma t_{1}} \\
\lambda^{B} & =\frac{(\Delta+2 \Gamma) p_{1}^{B}-(\Delta-\Gamma) p_{1}^{A}-3 \Gamma p_{1}^{U C}-\alpha_{1}\left(\alpha_{2}-\alpha_{1}\right) m}{6 \Gamma t_{1}}
\end{aligned}
$$


Suppose that both non-negativity constraint bind, then $p_{1}^{A}=-\theta, p_{1}^{B}=0$ and

$$
\begin{aligned}
\lambda^{A} & =\frac{-\theta(\Delta+2 \Gamma)-3 \Gamma p_{1}^{U C}+\left(3 \Gamma+\alpha_{1}\left(\alpha_{2}-\alpha_{1}\right)\right) m}{6 \Gamma t_{1}} \\
\lambda^{B} & =\frac{\theta(\Delta-\Gamma)-3 \Gamma p_{1}^{U C}-\alpha_{1}\left(\alpha_{2}-\alpha_{1}\right) m}{6 \Gamma t_{1}}
\end{aligned}
$$

This is an equilibrium if

$$
\begin{aligned}
\psi^{B}(m) & \leq \theta \leq \psi^{A}(m), \text { when } \Delta-\Gamma>0 \\
\theta & \leq \min \left\{\psi^{B}(m), \psi^{A}(m)\right\}, \text { when } \Delta-\Gamma<0 \\
\theta & \leq \psi^{A}(m) \text { and } 3 \Gamma p_{1}^{U C}+\alpha_{1}\left(\alpha_{2}-\alpha_{1}\right)<0 \text { when } \Delta-\Gamma=0 \\
\text { where } \psi^{A}(m) & =\theta^{A}+\rho m, \text { and } \rho=\frac{3 \Gamma+\alpha_{1}\left(\alpha_{2}-\alpha_{1}\right)}{\Delta+2 \Gamma} \\
\psi^{B}(m) & =\frac{3 \Gamma p_{1}^{U C}+\alpha_{1}\left(\alpha_{2}-\alpha_{1}\right) m}{\Delta-\Gamma} .
\end{aligned}
$$

Suppose now that $\lambda^{A}=0$ and $\lambda^{B}=0$. Solving the system we obtain the following equilibrium prices:

$$
\begin{aligned}
& p_{1}^{A *}(m)=p_{1}^{U C}-\left(\frac{\alpha_{1}\left(\alpha_{2}-\alpha_{1}\right)+\Delta+2 \Gamma}{2 \Delta+\Gamma}\right) m \\
& p_{1}^{B *}(m)=p_{1}^{U C}-\left(\frac{3 \Gamma-\alpha_{2}\left(\alpha_{2}-\alpha_{1}\right)}{2 \Delta+\Gamma}\right) m
\end{aligned}
$$

which can be solution if

$$
\theta \geq-p_{1}^{A *}(m) \text { and } p_{1}^{B *}(m) \geq 0
$$

Suppose now that $\lambda^{A}=0$ and $\lambda^{B}>0$, consequently $p_{1}^{B}=0$. Solving the system we obtain the following equilibrium prices:

$$
\begin{aligned}
p_{1}^{A} & =-\psi^{A}(m) \\
\lambda^{B} & =\frac{-(2 \Delta+\Gamma) p_{1}^{U C}+\left(3 \Gamma-\alpha_{2}\left(\alpha_{2}-\alpha_{1}\right)\right) m}{2 t_{1}(\Delta+2 \Gamma)}
\end{aligned}
$$

which is an equilibrium if

$$
\theta>\psi^{A}(m) \text { and } 0 \geq p_{1}^{B *}(m) .
$$

Suppose now that $\lambda^{A}>0$ and $\lambda^{B}=0$, consequently $p_{1}^{A}=-\theta$. Solving the system we obtain the following equilibrium prices:

$$
\begin{aligned}
p_{1}^{B} & =\frac{3 \Gamma p_{1}^{U C}+(\Delta-\Gamma)(-\theta)+\alpha_{1}\left(\alpha_{2}-\alpha_{1}\right) m}{\Delta+2 \Gamma} \\
\lambda^{A} & =\frac{-(2 \Delta+\Gamma)\left(\theta+p_{1}^{U C}\right)+\left(\alpha_{1}\left(\alpha_{2}-\alpha_{1}\right)+\Delta+2 \Gamma\right) m}{2 t_{1}(\Delta+2 \Gamma)}
\end{aligned}
$$


which is an equilibrium if:

$$
\begin{aligned}
\theta & <\min \left\{\psi^{B}(m),-p_{1}^{A *}(m)\right\}, \text { when } \Delta-\Gamma>0 \\
\psi^{B}(m) & <\theta<-p_{1}^{A *}(m), \text { when } \Delta-\Gamma<0 \\
\theta & <-p_{1}^{A *}(m) \text { and } 3 \Gamma p_{1}^{U C}+\alpha_{1}\left(\alpha_{2}-\alpha_{1}\right)>0, \text { when } \Delta-\Gamma=0
\end{aligned}
$$

Notice that:

$$
\begin{aligned}
\psi^{A}(m)-\psi^{B}(m) & =-\frac{3 \Gamma(\Gamma+2 \Delta)}{(\Delta+2 \Gamma)(\Delta-\Gamma)} p_{1}^{B *}(m) \\
\phi^{A}(m)-\psi^{B}(m) & =-\left(\frac{\Delta+2 \Gamma}{\Delta-\Gamma}\right) p_{1}^{B *}(m)
\end{aligned}
$$

Notice also that

$\phi^{B}(m)=-p_{1}^{U C}+\left(\frac{3 \Gamma-\alpha_{2}\left(\alpha_{2}-\alpha_{1}\right)}{2 \Delta+\Gamma}\right) m=-p_{1}^{U C}+\left(\frac{\Delta-\Gamma-\alpha_{1}\left(\alpha_{2}-\alpha_{1}\right)}{2 \Delta+\Gamma}\right) m .$.

Summing up, we have the following cases.

- First if $p_{1}^{B *}(m)>0$ and $p_{1}^{A *}(m)+\theta>0$, no price is constrained by the non-negativity constraint.

Otherwise:

- When $\Delta-\Gamma>0$ :

- When $p_{1}^{B *}(m)<0$, the price of the bundle is zero for $\theta \leq$ $\psi^{A}(m)$, and $p_{1}^{B}>0$ if $\theta<\psi^{B}(m)$.

- When $p_{1}^{B *}(m)>0$, the price of the bundle is zero and $p_{1}^{B}>0$.

- When $\Delta-\Gamma<0$ :

- When $p_{1}^{B *}(m)<0$, the price of the bundle is zero for $\theta \leq \psi^{A}(m)$, and $p_{1}^{B}=0$.

- When $p_{1}^{B *}(m)>0$, the price of the bundle is zero, and $p_{1}^{B}>0$ if $\theta>\psi^{B}(m)$.

- When $\Delta-\Gamma=0$, note that $\psi^{A}(m)=-p_{1}^{A *}(m)>0$. We thus have two cases:

- When $p_{1}^{A *}(m)>-\theta$, there are two regimes according to whether $p_{1}^{B *}(m)$ is positive or negative.

- When $p_{1}^{A *}(m)<-\theta$, there are two regimes according to whether $3 \Gamma p_{1}^{U C}+\alpha_{1}\left(\alpha_{2}-\alpha_{1}\right)$ is positive or negative. 
Proof of propositions 9 and 8. Platform's total profits are thus:

$$
\begin{aligned}
& \Pi_{P}^{A}(m)=\Phi\left(p_{1}^{A}(m), 0\right)+\frac{\alpha_{1}\left(-6 \Gamma+2 \sigma p_{1}^{A}(m)+\alpha_{1} m\right)}{18 t_{1} \Gamma} m \\
& \Pi_{P}^{B}(m)=\Phi\left(0, p_{1}^{A}(m)\right)+\frac{3 \Gamma\left(3 t_{1}-\alpha_{1}\right)-\left(8 \Gamma+\alpha_{1}\left(\sigma-\alpha_{1}\right)\right) p_{1}^{A}(m)+\alpha_{1}^{2} m}{18 t_{1} \Gamma} m
\end{aligned}
$$

where $\Phi(x, y)$ is defined in the section on tying. Recall that $\Pi_{T}^{A}(m)=$ $\Phi\left(p_{1}^{A}(0), 0\right)+m$. We thus have:

$$
\left.\frac{d\left(\Pi_{P}^{A}(m)-\Pi_{T}^{A}(m)\right)}{d m}\right|_{m=0}=\frac{\alpha_{1}\left(-6 \Gamma-2\left(\alpha_{2}-\alpha_{1}\right) \theta\right)}{18 t_{1} \Gamma}-1
$$

which is negative if $\alpha_{1} \leq \alpha_{2}$. Recall that $\Pi_{T}^{B}(m)=\Phi\left(0, p_{1}^{A}(0)\right)$. When $p(m)=p(0)$, we thus have:

$$
\left.\frac{d\left(\Pi_{P}^{B}(m)-\Pi_{T}^{B}\right)}{d m}\right|_{m=0}=\frac{3 \Gamma\left(3 t_{1}-\alpha_{1}\right)+\left(7 \Gamma+t_{1} t_{2}-2 \alpha_{1}^{2}\right) \theta}{18 t_{1} \Gamma}
$$

which is positive if $\alpha_{1}$ is small.

The equilibrium profits are still given by the above equations. The slopes $\left.\frac{d\left(\Pi_{P}^{j}(m)-\Pi_{T}^{j}(m)\right)}{d m}\right|_{m=0}$ are now augmented by $-\rho \Phi_{1}\left(p_{1}^{A}(0), 0\right)$ for $A$ and $-\rho \Phi_{2}\left(0, p_{1}^{A}(0)\right)$ for $B$.

Proof of proposition 10. The profit is:

$$
\begin{aligned}
& \Pi_{P}^{A}(m)=\Pi^{C}-\frac{1}{2 t_{1}} p_{1}^{A}(m)^{2}+\frac{\left(t_{1}+f_{1}-\alpha\right)}{2 t_{1}} p_{1}^{A}(m)+\frac{\alpha(-6 \Gamma+\alpha m)}{18 t_{1} \Gamma} m \\
& \Pi_{P}^{B}(m)=\Pi^{C}-\frac{f_{1}}{2 t_{1}} p_{1}^{A}(m)+\frac{3 \Gamma\left(3 t_{1}-\alpha\right)-\left(8 \Gamma-\alpha^{2}\right) p_{1}^{A}(m)+\alpha^{2} m}{18 t_{1} \Gamma} m
\end{aligned}
$$

For $\alpha$ small, the profit of platform $B$ increases with $m$ and decreases with $p_{1}^{A}$. Thus platform $B$ prefers the situation with pure bundling $(m>0)$ to tying $(m=0)$. Notice that interiority conditions $n_{i}^{A}<1$ imply that $m<\min \left\{\frac{3 \Gamma}{\alpha^{2}}\left(t_{1}+p_{1}^{A}(m)\right), \frac{3 \Gamma}{\alpha}\right\}$.

Using $p_{1}^{U C}=t_{1}+f_{1}-\alpha$ and $\alpha m<3 \Gamma$ we have:

$$
\Pi_{P}^{A}(m)<\Pi^{C}+\frac{1}{2 t_{1}}\left(p_{1}^{U C}-p_{1}^{A}(m)\right) p_{1}^{A}(m) .
$$

Since $p_{1}^{A}(m)=p_{1}^{A}(0)$ if $p_{1}^{A}(0)=-\theta$, we confirm also that platform $A$ prefers tying when it implies a free bundle. Moreover, in the case where tying implies a positive price, $p_{1}^{A}(0)=\frac{p_{1}^{U C}}{2}$, implying that the RHS is maximal in $p_{1}^{A}$. Thus platform $A$ prefers tying. 